\title{
ESTUDO DO COEFICIENTE DE TRANSFERÊNCIA DO VAPOR DE AGUA SOBRE CULTURA DE FEIJÃO
}

\author{
JERONIMO GARCIA VILLANUEVA
}

Orientador: Prof. Dr. KLAUS REICHARDT

Dissertação apresentada à Escola Superior de Agricultura "Luiz de Queiroz", da Universidade de São Paulo, para obtenção do título de Mestre em Agronomia, Área de Con centração: Agrometeorologia.

PIRACICABA

Estado de São Paulo - Brasil

Novernbro - 1987 
García Vellanueva, Jerónimo

G216e Estudo do coeficiente de transferência do va por de ägua søbre cultura de feijão. Piracicaba, 1987.

58p. ilus.

Diss. (Mestre) - ESALQ

Bibliografia

1. Feijão - Balanço de energıa. 2. Feijão Evapotranspiração. I. Escola Superior de Agricultura Luiz de Queiroz, Piracicaba.

CDD 635.652 
ESTUDO DO COEFICIENTE DE TRANSFERENCIA DO CAPOR DE AGUA SOBRE CULTURA DE FEIJÃO

JERONIMO GARCIA VILLANUEVA

Aprovado em: 17/03/1988

Comissão Julgadora:

Prof. Dr. Klaus Reichardt

ESALQ/USP

Prof. Dr. Nilson Augusto Villa Nova

ESALQ/USP

Dr. Jefferson Mortati

CENA/USP

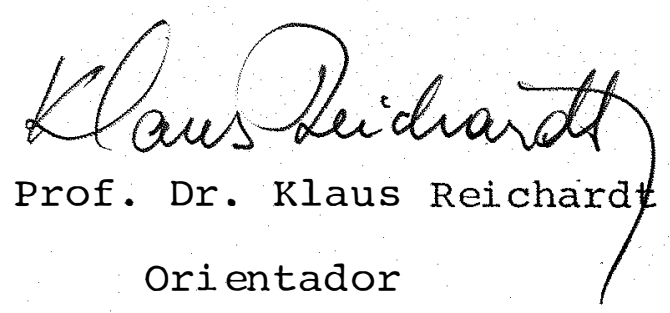


ii.

OFEREÇO:

A Deus que me encaminhou pela senda da antecipação e pela exatidão das minhas ações.

DEDICO:

Aos meus pais ANTONIO GARCIA M.

e MARTHA VI LLANUEVA L., Irmãos e Irmãs. 


\section{AGRADECIMENTOS}

Ao Dr. Kalus Reichardt, pelo apoio, incentivos; pela amizade e excelente orientação.

Ao Convênio UNA/BIRF, pelo apoio financeiro.

Ao Prof. Dr. Paulo Leonel Libardi, pela colaboração e apoio.

Ao Prof. Dr. Nilson Augusto Villa Nova pelas sugestões mais oportunas.

Ao Prof. Dr. Luiz Roberto Angeloci pelas sugestões.

Ao Prof. Dr. Epaminondas S.B. Ferraz, pela colaboração e apoio.

Ao Prof. Dr. Armando Bergamin Filho, do Departamento de Fitopatologia (ESALQ/USP), pela colaboração com equipamento meteorológico.

Ao Prof. Dr. Carlos Ometto pela amizade e colaboração.

A Antonio José Leite Ferraz pela colaboração.

A Ivan José Moretti pela amizade e colaboração na parte instrumental.

A Escola Superior de Agricultura "Luiz de Queiroz", da Uni versidade de São Paulo por haver me acolhido no Curso de Pós-Graduação de Agrometeorologia.

A todos os funcionários do Departamento de Física e Meteorologia da ESALQ/USP, que direta ou indiretamente contribuiram para minha formação. 
iv.

\section{INDICE}

Pāgina

LISTA DE TABELAS $\ldots \ldots \ldots \ldots \ldots \ldots \ldots \ldots \ldots \ldots \ldots \ldots \ldots \ldots \ldots$

vii

LISTA DE FIGURAS $\ldots \ldots \ldots \ldots \ldots \ldots \ldots \ldots \ldots \ldots \ldots \ldots \ldots \ldots$

viii

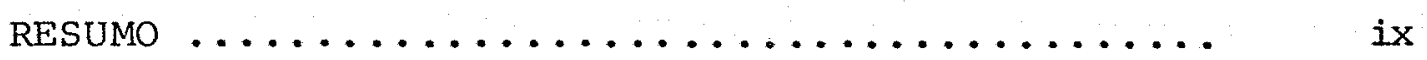

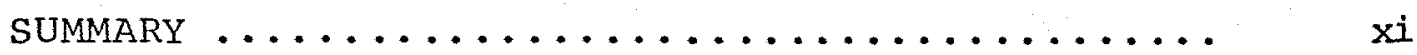

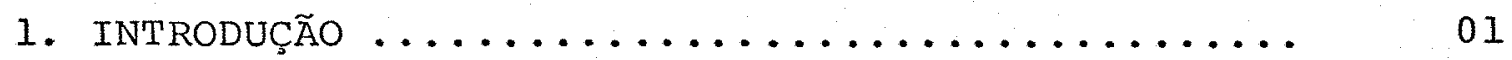

2. REVISÃo DE LITERATURA ........................ 03

2.1. Transferência vertical na atmosfera..... 03

2.2. Camada limite ................... 04

2.3. Coeficientes de intercâmbio e transferência

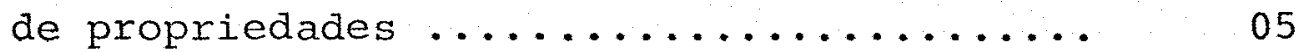

2.4. Formas de perfis do vento ............. 08

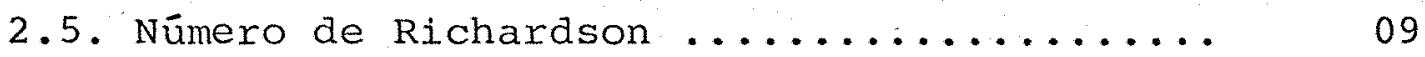

2.6. Coeficiente de transferēncia vertical do

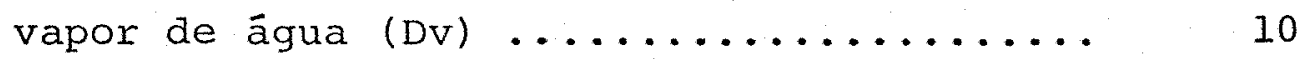

2.7. Fluxo do vapor de água segundo o método ae

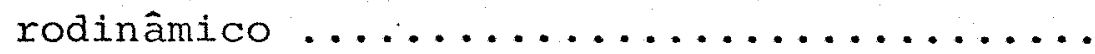

2.8. Fluxo do vapor de água segundo o método de

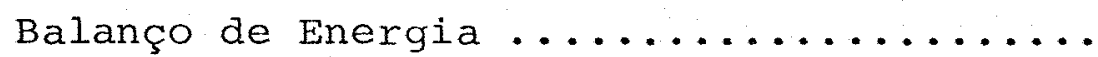

2.9. Fluxo do vapor de água segundo o método de

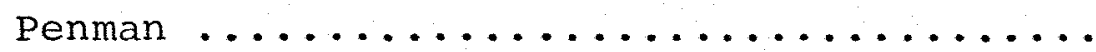

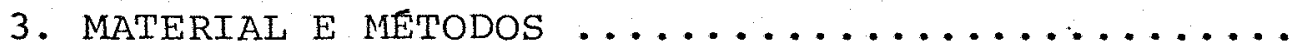

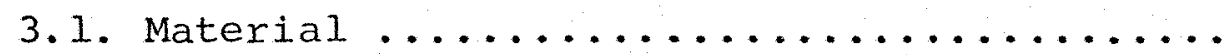

3.1.1. Características das condições expe-

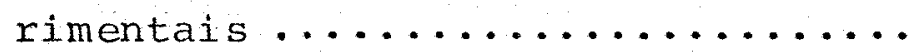

3.1.1.1. Local e solo ..........

3.1.1.2. Cultura e condições de ob-

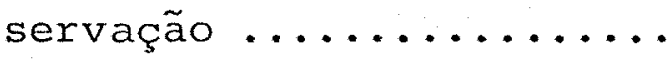


3.1.2. Instrumental $\ldots \ldots \ldots \ldots \ldots \ldots \ldots \ldots 18$

3.1.2.1. Radiômetro líquido ....... 18

3.1.2.2. Placas medidoras do fluxo de calor no solo ......... 18

3.1.2.3. Psicrômetros .......... 18

3.1.2.4. Anemômetros ........... 19

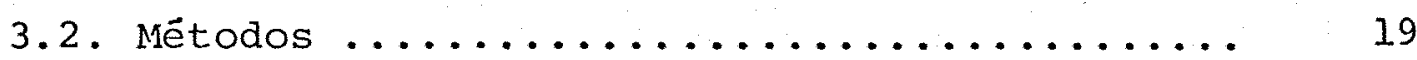

3.2.1. Psicrometria ............... 19

3.2.1.1. Construção e calibração ... 20

3.2.1.2. Determinação da umidade do ar .................... 21

3.2.2. Método de Balanço de Energia ....... 23

3.2.2.1. Razão de Bowen .......... 23

3.2.2.2. Energia de evapotranspira ção (LE) $\ldots \ldots \ldots \ldots \ldots \ldots \ldots \ldots$

3.2.2.3. Energia de aquecimento do ar $(H) \ldots \ldots \ldots \ldots \ldots \ldots \ldots \ldots \ldots$

3.2.3. Método Aerodinâmico ............ 24

3.2.3.1. Estabilidade atmosférica... 24

3.2.3.2. Número de Richardson (Ri) . 25

3.2.3.3. Função de estabilidade $(\phi)$. 26

3.2.3.4. Energia de evapotranspiração (LE) $\ldots \ldots \ldots \ldots \ldots \ldots \ldots \ldots \ldots \ldots$

3.2.3.5. Energia de aquecimento do $\operatorname{ar}(\mathrm{H}) \ldots \ldots \ldots \ldots \ldots \ldots \ldots$

3.2.4. Coeficiente de transferência do vapor de água (Dv) ............. 30

3.2.4.1. Quantificação ......... 30

3.2.4.2. Relações empíricas para estimativa de Dv .......... 32

3.2.4.2.1. Relações lineares simples 33

3.2.4.2.2. Relações lineares múlti-

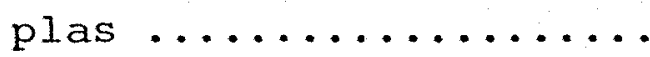


vi.

Pāgina

3.2. Tratamento dos dados do vento ..... 34

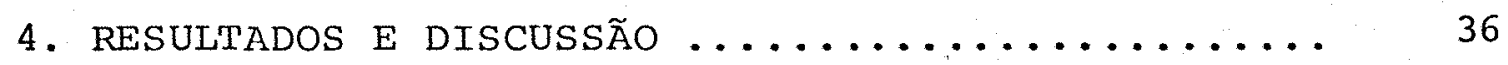

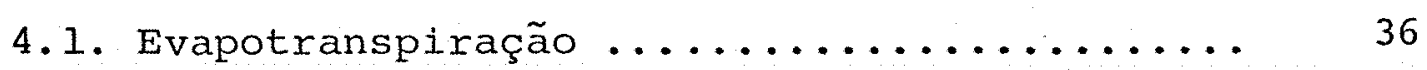

4.2. Coeficiente de transferência Dv .......... 42

4.3. Relações empíricas para estimativa de Dv .. 46

4.3.1. Relações lineares simples ........ 46

4.3.2. Relações lineares mültiplas ....... 49

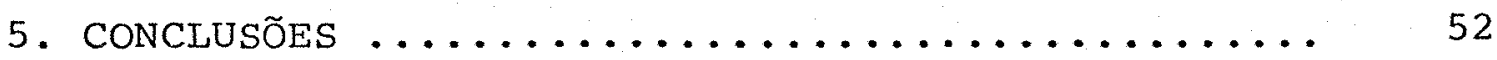

6. LITERATURA CITADA ................... 54

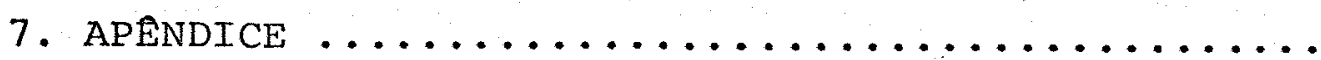




\section{LISTA DE TABELAS}

TABELA NQ

página

01 Totais diārias de radiação líquida $\mathrm{R}$ $\frac{\mathrm{Cal} .}{\mathrm{cm}^{2} \mathrm{dia}}$, fluxo do calor no solo $\mathrm{G}$ $\frac{\text { Cal. }}{\mathrm{cm}^{2} \text { dia }}$ e vento médio $(\mathrm{m} / \mathrm{s})$ a 40100

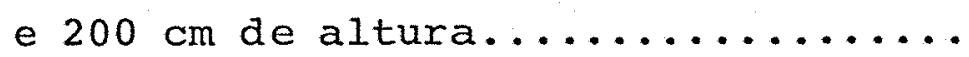

02 Valores diārios de evapotranspiração LE (mm/dia), calor sensivel $\mathrm{H} \frac{\mathrm{Cal} \text {. }}{\mathrm{cm}^{2} \mathrm{dia}}$ e valores médios diários do coeficien te de transferência Dv $(\mathrm{cm} / \mathrm{s}) \ldots . . .$.

03 Quadro de análise de variância para método de Balanço de Energia na camada $20-80 \mathrm{~cm} \ldots \ldots \ldots \ldots \ldots \ldots$

04 Quadro de análise de variância para método de Balanço de Energia na camada $20-140 \mathrm{~cm} \ldots \ldots \ldots \ldots \ldots$

05 Quadro de anālise de variância para método aerodinâmico na camada $20-80 \mathrm{~cm}$

06 Relações empíricas para estimativa do coeficiente de transferência do vapor

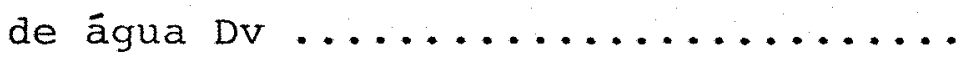


01 Variação horária do nūmero de Richardson $\mathrm{Ri}$ e evapotranspiração $\mathrm{LE} \frac{\mathrm{Cal}}{\mathrm{cm}^{2} \min }$ obtidos com método de Balanço de Energia e método Aerodinâmico (a); (b) con tinuação de (a) .................

02

Variação horāria do coeficiente de transferência do vapor de āgua (Dv) obtidos com dados experimentais e esti mados com método aerodinàmico (a); (b) e (c) continuação de (a) ............

03 Valores mëdios diários do coeficiente de transferência do vapor de água (Dv) obtidos com dados experimentais e estimados, com método Balanço de Energia (a), e método Aerodinâmico (b) ... 
ESTUDO DO COEFICIENTE DE TRANSFERENCIA DO VAPOR

DE đ̆GUA SOBRE CULTURA DE FEIJÃO

Autor: Jerōnimo García Villanueva Orientador: Prof. Dr. Klaus Reichardt

\section{RESUMO}

Desenvolveu-se um estudo experimental para quantificar o coeficiente de transferência vertical do vapor de āgua sobre cultura de feijoeiro, e pesquisar algumas relações empíricas para sua estimativa.

O coeficiente de transferência vertical foi avaliado usando dados experimentais da variação vertical de umidade $(\Delta e)$ e dados de evapotranspiração (LE) obtidos por método de Balanço de Energia e o método aerodinâmico. Para o estabelecimento das relações empiricas foram ensaiadas correla Ções lineares simples entre os valores horärios de DV e vento a dois metros acima da superfície; assim como correlações leneares mültiplas entre Dv médio e dados de umidade relativa; temperatura, temperatura māxima e mínima do posto meteorológi co.

Os resultados indicaram que os valores do coeficiente de transferência do vapor obtidos por método aerodinâmico foram maiores que os de método de balanço de energia; mas os valores nos dois casos estiveram dentro daqueles que 
podem dar-se na natureza. As correlações lineares simples resultaram ser altamente significativas nos dois métodos, embora comparando ambos os valores de FC e dos coeficientes de correlação $(\gamma)$ indicaram que o mētodo aerodinâmico foi o meIhor. A equação correspondente à camada $20-80 \mathrm{~cm}$ foi:

$$
\begin{aligned}
& \mathrm{DV}=2,582+1,238 \mathrm{~V} \\
& \mathrm{DV}=2,069+2,455 \mathrm{~V}
\end{aligned}
$$

para o mētodo de balanço de energia e método aerodinâmico, res pectivamente.

As correlações lineares múltiplas foram mais adequadas quando ensaiadas com temperatura $(T)$, umidade relativa (UR) e amplitude térmica diurna (AT) como variáveis inde pendentes e foram significativas a $90 \%$ de segurança, no caso do método aerodinâmico, com a equação seguinte:

$$
\mathrm{DV}=30,622+0,045 \mathrm{~T}-0,157 \mathrm{UR}-0,998 \mathrm{AT}
$$


VAPOR TRANSFER COEFFICIENT DETERMINATION ABOVE BEAN CANOPY AND HIS RELATIONSHIPS WITH OTHER METEOROGICAL DATA

Author: Jerónimo Garcị́a V. Adviser: Prof. Dr. Klaus Reichardt

\section{SUMMARY}

The hourly gradient of vapor pressure $(\Delta e)$ and evapotranspiration rate quantified by energy budget and aerodynamic methods was used to quantify the vertical vapor transfer coefficient (Dv) above a bean canopy during 23 days.

Values of Dv were used to determine simple linear relationships with wind velocity at a height of $2 \mathrm{~m}$. Multiple linear relationships with other meteorological data, were also studied.

Although the simples linear correlations, for both methods, were significant at $99 \%$ significancy level, the aerodynamic method was better than the energy budget method; the established relationships were:

$$
\begin{aligned}
& \mathrm{Dv}=2.582+1.238 \mathrm{~V} \text { and } \\
& \mathrm{Dv}=2.069+2.455 \mathrm{~V}
\end{aligned}
$$

respectively, for energy budget and aerodynamic method.

On the other hand, the multiple linear correlations were significant at $90 \%$ of significancy level, 
xii.

only for aerodynamic method, and the regression equation was:

$$
\mathrm{DV}=30.622+0.045 \mathrm{~T}-0.157 \mathrm{UR}-0.998 \mathrm{AT}
$$




\section{INTRODUCẼOO}

o conhecimento da perda de água por evapotrans piração de campos de cultura ou de diferentes superfícies naturais é de grande importância e necessário quando existe a necessidade de irrigação. Até agora têm sido desenvolvidos muitos métodos para sua estimativa; na maioria dos casos, a precisão dos resultados obtidos é boa quando aplicados em regiões com condições climáticas semelhantes às quais onde foram elaborados. Entre métodos de uso mais generalizado, temos o mëtodo de Penman, o método aerodinâmico e o método de balanço de energia. o método de penman é um dos mais comple tos; ele tem dois termos, o termo de energia e o termo aerodinâmico, sendo que o segundo precisa ser ajustado antes de ser utilizado. Existem muitos trabalhos sobre o termo aerodi nâmico, a maioria deles são estudos de correlação com dados de evaporímetro Piche. OMETTO (1981) estabelecar para Piracicaba o termo aerodinâmico como sendo $28 \%$ da evaporação do Piche. GARCIA (1984a) determina relações do termo aerodinâmico com radiação lí quida e amplitude térmica diuma, A precisão destas relaçōes obtidas estão na estreita dependência da expressão usada para estima- 
tiva do termo aerodinâmico e como é bem conhecido ela è função da velocidade do vento, além do défice de saturação do ar. Em localidades onde o fator altitude predomina, as equações usadas para sua estimativa não dão resultados bons (GARCIA, 1984b). Há portanto a necessidade de serem feitos estudos experimentais para determinar equações de relação entre o vento e o coeficiente de transferência vertical do vapor de água (Dv) para estabelecer um termo aerodinâmico correto para cada localidade.

O presente trabalho tem, portanto, o objetivo de desenvolver uma metodologia para determinar o coeficien te de transferência vertical do vapor de água sobre uma cultu彑 ra e pesquisar equações empiricas para sua estimativa. 


\section{REVISÃO DE LITERATURA}

\subsection{TRANSFERÊNCIA VERTICAL NA ATMOSFERA}

Os processos de transferência vertical entre a superficie da Terra e atmosfera se dão a nível molecular e por turbulência atmosférica. A transferência molecular é uma consequência direta da agitação molecular (velocidade média das moléculas de ar $485 \mathrm{~m} / \mathrm{s}$ ) a qual na presença de um gradiente vertical de uma certa propriedade gera uma troca constante de moléculas entre camadas horizontais adjacentes, produzin do transferência de momento, calor ou difusão de massa (MONTEITH, 1973). As transferências por turbulência são de natureza muito complexa; as porções do fluido ou parcelas do ar transferem momento e outras propriedades de uma camada a outra. o vento, nas proximidades de obstáculos, como é o caso nas proximidades do solo é altamente turbulento. o fluxo des te vento è de natureza complexa, é caracterizado pela existência de grande número de movimentos secundários transversais ao fluxo principal horizontal, produnzindo uma incessante mistura do ar; é um fluxo altamente rotacional, mas seus 
vörtices não têm estruturas comuns (SUTTON, 1953; HALTINER, 1957).

os processos de transferência moleculares são de grande importância na camada limite laminar, próxima ao solo, como nas superficies das folhas ou outras superficies com "rugosidades" menores. O transporte turbulento é devido ao fluxo rotacional do vento e é importante na camada limite turbulenta situada acima da laminar. Na camada limite laminar os processos de transferência molecular podem ser estimados utilizando os coeficientes de difusão médios, dados pela expressão:

$$
\frac{\mathrm{D}(\mathrm{T})}{\mathrm{D}(0 \circ \mathrm{C})}=\frac{\mathrm{k}(\mathrm{T})}{\mathrm{k}(0 \circ \mathrm{C})}=\frac{\mathrm{V}(\mathrm{T})}{\mathrm{V}(0 \circ \mathrm{C})}=(1+0,007 \mathrm{~T}) \text {, } \mathrm{T} \text { em oC }
$$

onde o coeficiente de difusão de vapor de água à zero graus centigrados, $D(00 \mathrm{C})$ é igual a $0,212 \mathrm{~cm}^{2} / \mathrm{s}$ do calor sensível $\mathrm{k}(0 \circ \mathrm{C})$ é igual a $0,189 \mathrm{~cm}^{2} / \mathrm{s}$ e o coeficiente de difusão molecular de momento ou coeficiente de viscosidade cinemá tica a zero graus v(00C) é igual a $0,133 \mathrm{~cm}^{2} / \mathrm{s}$ (MONTEITH, 1973).

\subsection{Camada limite}

E uma zona na qual a velociade horizontal de um fluxo sobre obstāculos aúmenta com a altura, ou seja, è função da altura (z). Existem dois tipos de camada limite, 
a laminar e a turbulenta. Quando as linhas de corrente do fluxo são praticamente paralelas tem-se a camada limite laminar.

Nesta camada as trocas de mómento e outras pro priedades realizam-se por processos moleculares. A espessura desta camada é muito variável. Quando tratar-se de super fícies lisas ou de pequena rugosidade, a camada limite começa sendo laminar e é nestes casos que ela tem maior espessura; ao aumentar, o fluxo converte-se em fluxo turbulento, destru indo-se a camada limite laminar, dando lugar à camada limite turbulenta onde o movimento é muito desordenado sem padrões definidos.

\subsection{COEFICIENTES DE INTERCÂMBIO E TRANSFERÊNCIA DE PROPRIEDADES}

Segundo SCHMIDT (SUTTON, 1953) a transferência de qualquer uma das propriedades conservativas pode ser expressa em termos de coeficientes virtuais (de viscosidade, condutivida de, dịfusividade) denominados como coeficientes de intercâm bio. Sendo E uma propriedade conservativa em transferência por unidade de volume, em um fluxo horizontal turbulento, o transporte vertical ou fluxo vertical médio (F) da proprieda de E, por unidade de área e tempo é dada pela equação:

$$
F=-\left(k \frac{d \bar{E}}{d z}+\rho \overline{E^{\prime} W}\right)
$$


na qual o produto das flutuações da propriedade $E$ e da velocí dade $W$ designado por $\overline{E^{\prime} W}$ ' è conhecido. Segundo a hipótese dos coeficientes de intercâmbio o movimento turbulento pode ser tratado de acordo com a teoria cinética dos gases, onde a transferência de qualquer propriedade conservativa pode ser expressa por meio de coeficientes virtuais de viscosidade, condutividade, difusividade, multiplicados por gradientes do valor médio da propriedade en transferencia. Assim, denominando A o coeficiente virtual, a equação do fluxo médio da propriedade E, fica:

$$
F=-\frac{d \bar{E}}{d z}(k+A)
$$

e a transferência vertical molecular e turbulenta do momento, considerando $\overline{\mathrm{E}}=\overline{\mathrm{u}}, \mathrm{k}=\mathrm{p}, \mathrm{v}, \mathrm{A}=\mathrm{p} \mathrm{Km}$ e $\mathrm{F}=\tau$, fica:

$$
\tau=\rho \frac{d \bar{u}}{d z}(v+K m)
$$

onde $v$ é o coeficiente de transferência molecular (viscosidade cinemátical em $\mathrm{cm}^{2} / \mathrm{s}$ para o caso do calor sensivel, considerando $\bar{E}=\bar{\theta}$ (temperatura potencial), $k=C p \cdot \rho \mathrm{kh}$, $A=\rho$ CpKh e $F=H$, fica na forma:

$$
H=-\rho C p \frac{d \bar{\theta}}{d z}-(k h+k h)
$$

sendo kh o coeficiente de difusão molecular de calor ou difusividade térmica $\left(\mathrm{cm}^{2} / \mathrm{s}\right)$. De maneira semelhante para caso 
de transferência vertical de massa de vapor de água, a propriedade conservativa a ser usada è a unidade especifica $(E=\bar{q})$, - coeficiente de intercâmbio $\mathrm{A}=\rho \mathrm{KV}$ e o coeficiente de trans ferência molecular $\mathrm{k}=\rho \mathrm{kv}$, então:

$$
\mathrm{E}=-\rho \frac{\mathrm{d} \overline{\mathrm{q}}}{\mathrm{dz}}(\mathrm{kv}+\mathrm{Kv})
$$

sendo $\mathrm{Km}$, $\mathrm{Kh}$ e $\mathrm{KV}$, respectivamente, o coeficiente de difusivi dade turbulenta para momento, para calor e vapor de água $\left(\mathrm{cm}^{2} /\right.$ s) (SUTTON, 1953; HALTINER, 1957; MONTEITH, 1973).

A aplicabilidade destas equações está condicionada ao conhecimento dos coeficientes de difusividade turbulenta de cada propriedade. Podemos dizer que existem três formas de tratar este problema: (1) Considerar que os coeficientes de difusividade turbulenta são iguais aos de uma atmosfera de estabilidade neutra; (2) considerar os coeficien tes de difusividade para calor e vapor diferentes do $\mathrm{Km}$, que é o caso mais geral ou caso de atmosfera instável e (3) deter minar o coeficiente de transferência de vapor de água (Dv) e de calor (Dh) mediante trabalhos experimentais.

SVERDRUP (HALTINER, 1957), indica que nas proximidades da superfície, quando a turbulência é determinada pe la natureza da superfície, isto è, turbulência forçada ou mecânica, os coeficientes de difusividade turbulenta são iguais entre si. Isto não acontece, porēm, quando predomina turbulência de convecção livre na qual, segundo MONTEITH (1973) o coeficiente de difusividade turbulenta de calor (Kh) è maior 
do que do vapor de água $(\mathrm{Kv})$. Assim quando $\mathrm{Km}=\mathrm{Kh}=\mathrm{Kv}$, 0 problema estará resolvido com a determinação de $\mathrm{Km}$, o que se consegue recorrendo ao perfil logaritmico do vento, que tem validade só para condições de atmosfera quase neutra, ou seja, quando não existem forças ascencionais ou turbulência tēr $\mathrm{mica}$.

Na maioria dos casos, na proximidade da super fície, durante um dia com vento fraco, a convecção livre predomina sobre a convecção forçada, mas em geral os dois proces sos coexistem durante o dia, o que é chamado de convecção mis turada. Nestas situações a presença da conveção livre serve para amplificar os "eddies" ou rodamoinhos da convecção força da gerados pelo vento cortante vertical $\frac{\partial u}{\partial z}$, amplificando ou intensificando grandemente os processos de transferência vertical.

\subsection{Formas DOS PERFIS DO VENTO}

Em um processo de plena convecção forçada, a estrutura dos "eddies" gerados pelo vento cortante, não são afetados por amplificações ou destruições significativas e po de-se considerar que a camada limite está em estabilidade neụ tra e o perfil do vento é do tipo logarítmico. Em condições instáveis ou de gradiente, os movimentos verticais são amplia dos pelo empuxo ("bouyancy") com um aumento que é inversamen te proporcional ao vento cortante existente; nelas as flutua- 
ções da velocidade vertical (W') è maior que a flutuação da velocidade horizontal $\left(u^{\prime}\right)$. No caso de atmosfera estável ocor re o processo inverso e a eqquação geral do perfil do vento é da forma:

$$
\frac{\partial u}{\partial z}=\frac{u^{*}}{k(z-d)} \phi_{m}
$$

onde $\phi_{\mathrm{m}}$ é a função da estabilidade (MONTEITH, 1973; BAILEY, 1980) ou termo de correção adiabātica que è um termo adicional, tendo seu valor igual a 1 em atmosfera neutra, maior que 1 em atmosfera estável e menor de 1 em condições instáveis.

\subsection{Número DE RichaRdson (RI)}

A utilização do perfil logaritmico de vento pa ra a estimativa do coeficiente de difusividade turbulenta de momento (km), implica no conhecumento prévio das condições em que foram feitas as medições, se em atmosfera neutra, ou se só houve movimento turbulento forçado. O nümero de Richardson permite reconhecer se o fluxo foi turbulento forçado ou de con vecção livre. O Ri é um parâmetro adimensional e, assumindo que os coeficientes de difusividade turbulenta de calor e de momento sejam iguais, ele representa a taxa de produção de energia térmica turbulenta em relação a taxa de produção de energia turbulenta atravēs do cisalhamento do vento, ou seja, expressa a razão entre os regimes de turbulência livre e 
forçada, ou ainda o quociente entre forças convectivas e forças mecânicas (ROSENBERG, 1974; ANDRE, 1981). O Ri é positivo sob condições de inversão (estabilidade) ruando a turbulência tende a desaparecer, e é negativo em condições instáveis, quando a turbulência tende a aumentar. Em condições de atmosfera neutra, Ri é igual a zero (CHANG, 1974, ROSENBERG, 1974). De acordo com TANNER (1963) e YAMAMOTO (1975), o Ri é função da altura em que é determinado, assim como não existe um Ri crítico (māximo).

\subsection{COEFICIENTE DE TRANSFERÊNCIA VERTICAL DE VAPOR DE AGUA (DV)}

Segundo SELLERS (1967) e BUDYKo (1956) este coeficiente é um termo que resulta da integração da equação de fluxo vertical médio do vapor de água da superfície evaporan te $\left(z_{0}=0\right)$ até outro nível $z$ que geralmente é $2 \mathrm{~m}$ acima do solo, ou seja:

$$
\begin{gathered}
E_{0}=\rho D_{V}\left(q_{0}-q_{z}\right)=\frac{\rho \varepsilon_{p} D_{V}}{F}\left(e_{0}-e_{z}\right), \text { onde } \\
D_{V}=\left(\int_{0}^{z} \frac{1}{k_{v}+k_{V}} d z\right)^{-1} \text { é coeficiente de transferência verti- } \\
\text { cal do vajpor de āgua }(\mathrm{cm} / \mathrm{s}) . \quad \text { Estes }
\end{gathered}
$$

autores indicam que este coeficiente é uma função da velocida de horizontal do vento, na forma de uma relação linear: $D_{v}=a+b u$. Neste caso o transporte vertical do vapor de 
água de superfícies aquáticas ou superfícies bem irrigadas è igual a $E_{0}=\rho(a+b u)\left(q_{0}-q_{z}\right)$ ou $E_{0}=\frac{\varepsilon \rho}{P} \cdot(a+b u)\left(e_{0}-e_{z}\right)$ Alēm disso afirma-se que ò coeficiente $D_{V}$ tem uma variação muito pequena na camada de um ou dois metros acima da superfície do solo.

\subsection{Fluxo do vapor de Agua segundo o MÉtodo AERODINÂMI CO}

Thornthwaite e Holzman (CHANG, 1974; OMETTO, 1981) desenvolveram este método para a estimativa da evapotranspiração sobre vegetações de porte baixo. Nestas a evapotranspiração (E) é função direta das umidade específica $q_{2}$ ' $q_{1}$ e do vento $u_{2}, u_{1}$ nos níveis $z_{2}$ e $z_{1}$ respectivamente. Além disso, ela é afetada na forma inversa pelo logarítmo natural das alturas $z_{2} \odot z_{1}:\left(\ln z_{2} / z_{1}\right)^{2}$. PASKILL (1950) adaptou o método para culturas de maior porte, introduzindo o parámetro de deslocamento zero (d) o qual é um nîvel de altura na qual começa o transporte turbulento. A estimativa do fluxo do vapor de água e do fluxo de calor sensível por este método, exige a utilização de técnicas micrometeorológicas e assim requer, simultaneamente, perfís de velocidade do vento, perfís de temperatura e perfís de umidade do ar, todos eles medidos em alturas idênticas. A dedução deste método baseia-se no princípio ou hipötese da similaridade (CHANG, 1974; THOM, 1975) na qual os coeficientes de difisuvidade tur 
bulenta $K_{m}, K_{v}$ e $k_{h}$ são considerados iguais e, nestas condições, espera-se que os perfis de umidade, de temperatura e de vento sejam similares nas camadas próximas à superfície do so 10. Isto porém só ocorre em condições de atmosfera neutra. Em atmosfera moderadamente instável, na altura de dois metros, $\mathrm{K}_{\mathrm{h}}$ pode ser até duas vezes maior que $\mathrm{K}_{\mathrm{m}}$ e esta diferença tende a aumentar com altura acima do solo e com o grau de instabilidade. O inverso ocorre em condições de atmosfera es tável.

RIDER (1954), na anālise de 50 perfís apresenta que $\frac{\mathrm{K}_{\mathrm{h}}}{\mathrm{K}_{\mathrm{V}}}=1,14 ; \frac{\mathrm{K}_{\mathrm{h}}}{\mathrm{K}_{\mathrm{m}}}=1,48$ e $\frac{\mathrm{K}}{\mathrm{K}_{\mathrm{m}}}=1,23$. Testes exaustivos deste método no lago Hefner produziram resultados não satisfatórios (CHANG, 1974). PRUIT (1963) também mostrou que os resultados obtidos por este mētodo apresentaram grandes di ferenças dos obtidos com lisimetros. Todos estes resultados indicam que a hipōtese da similaridade só deve ser aplicada para condições de atmosfera neutra.

DEACON et alii (1958) indicaram que este mētodo fornece bons resultados para cultura de porte baixo, sob certas condições, mas para culturas como trigo, cana-de-açū car, etc. não deve ser aplicado devido ao fato de ser cul turas de porte alto. A equação de PASKILL (1950) permite meIhores estimativas com este método, em comparação à equação de Thornthwaite-Holzman. Outra dificuldade para o caso de cultivos de porte alto é a determinação dos níveis adequados para a instalação dos instrumentos. Se as leituras forem 
realizadas muito prôximo à superfície, elas não serão representativas, uma vez que a superfície sempre é irregular; por outro lado, se as leituras forem feitas às alturas considerāveis, as diferenças entre os coeficientes de difusividade tur bulenta de vapor de água e momento podem ser muito grandes.

\subsection{Fluxo de vapor de agua segundo o método do BALANGO DE ENERGIA}

O método que utiliza a razão de BowEN para cál culo dos fluxos de vapor de água e calor sensivel é chamado có mo método de balanço de energia (VILLA NOVA, 1973) e também é conhecido por outros como método da razão de BOWEN (MONTHEIT, 1973, тном, 1975). Este método baseia-se no princípio de conservação de energia e é derivado da equação de balanço de energia. Para o caso do fluxo de vapor de água, sua expressividade é: $E=\left(R_{n}-G\right) / L(1+\beta)$. Para utilização deste método precisa-se fazer medições da radiação líquida $\left(R_{n}\right)$, do flu xo do calor do solo (G), como também da temperatura e da umi dade do ar em dois níveis. Estes últimos com a finalidade de estimar a razão de BOWEN ( $\beta$ ). A razão de BOWEN é a relação entre o fluxo turbulento de calor sensível (H) e o fluxo de vapor de água (E), dada pela equação $\beta=\gamma \frac{K_{h}}{K_{V}} \cdot \frac{\left(T_{0}-T_{1}\right)}{\left(e_{0}-e_{l}\right)}$. Da mesma forma como no método aerodinâmico, é utilizada a hipótese de similaridade, assumindo a igualdade entre os coeficientes de difusividade $\mathrm{K}_{\mathrm{h}}$ e $\mathrm{K}_{\mathrm{v}}$. O método de balanço de ener gia é menos sensivel às incorreções devidas aos coeficientes 
de difusividade turbulenta em comparação à equação de Tornthwaite-Holzman (CHANG, 1974). Quanto à razão de BOWEN é peque na, o erro na sua determinação terá efeitos pequenos na estimativa da evapotranspiração. TANNER (1960) tem mostrado que ele falha só para valores menores que $-0,5$. Para culturas bem irrigadas a razão de BOWEN, durante o dia, é geralmente de 0,1 . quando a evapotranspiração é alta. A noite, ao nascer e pôr do sol, quando a evapotranspiração aproxima-se ao valor do fluxo de calor sensivel, podem ocorrer grandes erros na es timativa da evapotranspiração.

\subsection{Fluxo de vapor de agua segundo o método de Penman}

Este método è uma combinação do método de balanço de energia com o método a erodinâmico, conseguinào desta forma eliminar a necessidade de realizar mediçôes a nivel de superfície (THOM, 1955; CHANG, 1974; THORNTWAITE, 1965). Sua equação original tem a forma:

$$
L E_{0}=\left[\frac{\Delta}{\gamma}\left(R_{n}-G\right)+f(u)\left(e_{S}-e\right)\right]\left(\frac{\Delta}{\gamma}+1\right)^{-1}
$$

onde: $R_{n}$ è a radiação líquida; $G$, fluxo de calor no solo; $\gamma$, constante psicrométrica; $\Delta$, inclinação da curva de satu ração; $\left(e_{S}-e\right)$ defice de saturação; $f(u)$, função de ven to.

A equação tem doìs termos, um chamado de termo energia $\left(R_{n}-G\right)$ e outro de termo aerodinâmico $E_{a} f(u)\left(e_{S}-e\right)$. o termo aerodinâmico è geralmente pequeno em comparação ao termo de energia mas tem variações muito grandes (STANHILL, 1962). $f(u) \bar{~}$ uma função da velocidade do vento, determinada empiricamente me 
diante observações da evaporação ( $E_{0}$ ) em superfícies de água livre, correlacionadas com a velocidade do vento $(u)$. Sua equação original para superfícies aquáticas foi $0,35(1+\mathrm{v} / 100)$ mas que, com os resultados de estudos feitos no lago Hefner, foi modificada para $0,35(0,5+u / 100)$, PENMAN (1956).

o uso deste mëtodo de estimativa da evapotrans piração necessita de observações de temperatura, umidade do ar a nivel do abrigo meteorológico, vento a dois metros acima do solo, da radiação líquida e fluxo do calor do solo.

$\mathrm{Na}$ maioria dos casos surgem problemas com o termo aerodinamico ( $\mathrm{E}_{\mathrm{a}}$ ) em consequência de seu envolvimento com a equação empirica da velocidade do vento. Desta forma foram feitos muitos estudos deste termo, existindo hoje em dia diferentes equa ções para sua estimativa. Também foram encontradas boas rela ções com dados de evaporímetro piche (Pi), amplitude térmica diurna, etc, como pode ser visto a seguir:

$$
\begin{aligned}
& E_{a}=0,1118+0,1469 \mathrm{E}-\text { segundo STANHILL }(1982) \\
& \mathrm{E}_{\mathrm{a}}=0,28 \mathrm{Pi} \\
& \mathrm{E}_{\mathrm{a}}=\left(0,26+0,26 \mathrm{C}_{\mathrm{t}} \mathrm{u}\right)\left(\mathrm{e}_{\mathrm{s}}-\right.\text { e) OLDEMAN \& FRERRE (198 } \\
& \mathrm{E}_{\mathrm{a}}=(0,35+0,0035 \mathrm{u})\left(\mathrm{e}_{\mathrm{s}}-\mathrm{e}\right) \text { COCHEME \& FRANQUIN }
\end{aligned}
$$

Segundo GILBERT \& VAN BAVEL (1954) a equação de Penman não produz estimativas a nível diärio; geralmente a boa precisão se dá para períodos maiores que 5 dias. VILLA NOVA (1973) apresenta uma equação modificada de Penman para estimativa da evapotranspiração a nível horário, na qual o termo aerodinâmico é estimado em função de dados meteorológicos me- 
didos no posto meteorológico padrão. A equação é a seguinte:

$$
\mathrm{E}_{\mathrm{a}}=(469,4 \overline{\mathrm{u}} . \Delta \mathrm{e})\left[\overline{\mathrm{T}}(174 \mathrm{q}+287)\left[\operatorname{Ln}\left(\frac{\mathrm{z}-\mathrm{d}}{\mathrm{z}_{\mathrm{o}}}\right)\right]^{2}\right]^{-1}
$$

onde $\bar{u}$ é velocidade do vento, média horária, à altura de $z=10 \mathrm{~m}$ expressa em $\mathrm{cm} / \mathrm{s} ; \Delta$ e é o défice de saturação, média horāria, medida no abrigo; $\overline{\mathrm{T}}$ é a temperatura do ar, média horāria, medida no abrigo $\left({ }^{\circ} \mathrm{K}\right)$; q é a umidade específica do ar $\frac{\mathrm{g} \cdot \mathrm{vapor} \text { de água }}{\mathrm{g} \text { ar úmido }}$ d è o plano de deslocamento zero e $z_{0}$ é a rugosi dade da superfície $(\mathrm{cm})$. O autor indica que a equação modifí cada só é aplicável a condições de evaporação potencial. 


\section{MATERIAL E MÉTODOS}

\subsection{Material}

3.1.1. Características das condições experimentais 3.1.1.1. Local e solo

o experimento foi conduzido na área experimental do Departamento de Física e Meteorologia da Escola Superios de Agricultura "Luiz de Queiroz", Piracicaba-sP $\left(22^{\circ} 43^{\prime} \mathrm{s}\right.$, $47^{\circ} 38^{\prime} \mathrm{W}$ e altitude $\left.576 \mathrm{~m}\right)$; o ensaio ocupou uma extensão de $20 \times 30 \mathrm{~m}$. O solo da ārea foi classificado como Terra Roxa Estruturada, série Luiz de Queiroz (RANZANI et alii, 1966).

3.1.1.2. Cultura e condições de observação

o experimento foi conduzido em cultura de fei-jão (Phaseolus vulgaris). O plantio, em linhas, foi realizado em 13/03/87. O solo foi mantido sempre em condições de água disponível próxima à capacidade de campo. Começaram-se as observações após 15 dias $(27 \% 03 / 87)$. A cobertura e a altura da cultura teve muita variação ate a etapa do início da floração $(17 / 04 / 87)$, estágio no qual a altura mẹ 
dia alcançada foi de $35 \mathrm{~cm}$. Após a etapa de plena floração, a cobertura e crescimento da cultura permaneceu quase constante, sendo em média de $40 \mathrm{~cm}$.

\subsubsection{Instrumental}

\subsubsection{Radiômetro Líquido}

Foi utilizado um radiômetro líquido inflável, tipo Funk, marca Middleton, com constante $28,173 \mathrm{mv} / \mathrm{cal} . \mathrm{cm}^{-2}$. $\min ^{-1}$. Instalou-se sobre um tripé a uma altura de $40 \mathrm{~cm}$ do topo da cultura.

3.1.2.2. Placas medidoras de fluxo de calor no solo

o fluxo de calor no solo foi medido com duas placas de constante igual a $16,80 \mathrm{mV} / \mathrm{cal} \cdot \mathrm{cm}^{-2} \cdot \mathrm{min}^{-1}$, instala das a aproximadamente $1,5 \mathrm{~cm}$ de profundidade, uma no solo com cobertura e outra no solo sem cobertura, na entrelinha.

\subsubsection{Psicrômetros}

Foi utilizado um conjunto de 6 psicrômetros com sensores de termistores de diâmetro de $8 \mathrm{~mm}$ por $1,5 \mathrm{~mm}$ de espessura. Foram instalados a $0,10,20,40,80$ e $142 \mathrm{~cm}$ acima da superfície do solo, montados em suportes de tubo de PVC branco de $21 \mathrm{~mm}$ de diâmetro, no qual os sensores estiveram protegidos da radiação solar direta. Como não havia sistema de ventilação, os sensores correspondentes aos bulbos úmidos 
foram molhados 15 minutos antes de cada leitura. As leituras da resistência da cada termistor foram feitas utilizando ohmi metro de um multimedidor eletrônico digital MIC-2200A e com um potenciômetro foram feitas as leituras do radiômetro e das placas de fluxo de calor no solo.

\subsubsection{Anemômetros}

Foram utilizados anemômetros tipo caneca, marca Fuess montados sobre um mastro, a 40,100 e $160 \mathrm{~cm}$, acoplados, cada um deles, a um registrador elétrico, no qual regís trou-se as velocidades acumuladas de cada $100 \mathrm{~m}$ para os niveis de 40 e $100 \mathrm{~cm}$ e as velocidades acumuladas cada $500 \mathrm{~m}$ pa ra o nível de $160 \mathrm{~cm}$. O vento a $2 \mathrm{~m}$ foi registrado com um anemômetro marca Clima L.N. 4.3900.10. Os dados de umidade e temperatura do ar, radiação líquida e fluxo do calor no so1o foram obtidos cada hora, desde 8:00 horas até 17:00 horas, durante 28 dias.

\subsection{METODOS}

\subsubsection{Psicrometria}

Para medir o gradiente de umidade nas diferentes camadas do ar foram construídos psicrômetros de termistores. Sua construção e calibração realizou-se no laboratório do Departamento de Física e Meteorologia da ESALQ/USP. 


\subsubsection{Construção e calibração}

Empregaram-se termistores caracterizados

no

mercado como termistores de 170 a 180 ohms de resistência (tem peratura ambiente de 209C). Formaram-se dois conjuntos de circuitos em paralelo com 6 termistores cada, os quais foram dispostos em um tubo de PVC branco (21 mm de diâmetro) de $1,50 \mathrm{~m}$ de comprimento, com os sensores colocados aos níveis nos quais foram utilizados. A calibração consistiu em fazer leituras simultâneas da temperatura da água com o termistor (valor em ohms) e com um termômetro de mercúrio com escala em décimos de grau célcius (0 a 509C). A temperatura da água foi variada de $2 \% \mathrm{C}$ até $499 \mathrm{C}$ e fizeram-se 35 leituras com cada termistor. Com o conjunto de pares de dados de temperatura e de resistência se buscou as constantes das equações teóricas seguintes:

$$
\begin{aligned}
& R=A e^{B / T} \\
& R=A e^{T / B}
\end{aligned}
$$

cujas formas linearizadas são:

$$
\begin{aligned}
& \mathrm{T}=1 /(\mathrm{a}+\mathrm{b} \text { In } \mathrm{R}) \\
& \mathrm{T}=\mathrm{a}+\mathrm{b} \text { Ln } \mathrm{R}
\end{aligned}
$$

A título de ilustração mostramos a seguir 
coeficiente de correlação e o erro relativo médio (Er) para dois termistores:

\begin{tabular}{|c|cc|cc|}
\hline \multirow{2}{*}{ Termistor } & Erro médio (Er) & Coef. correlação $(r)$ \\
\cline { 2 - 5 } & Eq. 3 & Eq. 4 & Eq. 3 & Eq. 4 \\
\hline 1 & 0,046 & 0,072 & $-0,9999$ & $-0,9998$ \\
2 & 0,0949 & 0,0967 & $-0,9997$ & $-0,9997$ \\
\hline
\end{tabular}

o quadro mostra que a equação tem maior precisão que a (4), mas as diferenças não são significativas, podendo-se afirmar que ambas equações são muito boas. por esta razão optou-se pela equação (2) na forma (4) para a deter minação das temperaturas. Estabeleceram-se para cada termis tor sua própria equação, 12 no total. Na calibração dos 12 termistores achou-se um erro mäximo de $0,141 \%$ e um erro míni mo de $0,069 \%$ e o erro médio dos 12 termitores foi de $0,11 \%$

\subsubsection{Determinação de umidade do ar}

Com a leitura dos termitores e aplicação da equação (4) foram obtidas as temperaturas de bulbo seco e de bulbo úmido (Th), com as quais determinaram-se as pressões de vapor de água (e) utilizando a equação psicromētrica:

$$
e=e_{s h}-\frac{C_{p} P}{\varepsilon L}\left(T-T_{h}\right)
$$


onde $\mathrm{P}$ é a pressão atmosfêrica médias dos meses de abril e maio, igual a $955.52 \mathrm{mb} ; \mathrm{C}_{\mathrm{p}}$ é o calor específico do ar seco à pressão constante, igual a $0,24 \mathrm{cal} / \mathrm{g}^{\circ} \mathrm{K} ; \mathrm{e}_{\mathrm{sh}^{\prime}}$ pressão de sa turação à temperatura do bulbo úmido $(\mathrm{mb}) ; \varepsilon$ é uma constante igual a 0,622 ; L è o calor latente de mudança de fase de água líquida para o vapor, dade pela equação:

$$
L=(597,3-0,56 \mathrm{~T}) \mathrm{cal} / \mathrm{g}
$$

na qual $T$ é a temperatura de bulbo seco em oc. Como $C_{p} P / \varepsilon L$ é chamado constante psicrométrica e considerando que durante o experimento a temperatura mais alta ocorrida foi de $360^{\circ} \mathrm{e}$ a mais baixa de $120 \mathrm{C}$, vemos que à constante psicromêtrica oscí lou entre 0,639 a 0,624 .

Para calcular a pressão de saturação (e ${ }_{\text {sh }}$ ) primeiramente foram comparados os valores obtidos com a equa ção de TETENS, com a deduzida por GARCIA (1984) mediante a integração da equação de CLAUSIUS-CLAPEYRON e com a equação utilizada na elaboração da tabela de pressão de saturação so bre ãgua líquida em Smithsonian Meteorological Tables. Usando-se temperaturas entre $100 \mathrm{C}$ e $400 \mathrm{C}$ encontrou-se que a equa Ção de TETENS apresentou valores mais baixos e os mais altos corresponderam à equação mostrada no Smithsonian Mteorologi cal Tables, mas como esta equação é muito complexa, adotou se a segunda equação, que foi a seguinte

$$
e_{S}=A \cdot T^{b} \cdot e^{C / T}
$$

onde:

$$
\begin{aligned}
& A=9,4216 \times 10^{23} \\
& b=-5,07712
\end{aligned}
$$




$$
\begin{aligned}
& c=-6801,2693 \\
& e=\text { base dos logaritmos naturais } \\
& T=\text { temperatura em oK }
\end{aligned}
$$

3.2.2. Método de balanço de energia

\subsubsection{Razão de Bowen}

Sua equação original è da forma:

$$
\beta=\gamma \frac{K_{h}}{K_{v}} \frac{\left(T_{1}-T_{2}\right)}{\left(e_{1}-e_{2}\right)}
$$

sendo $\gamma=C_{p} P / \varepsilon L$, a constante psicrométrica; $K_{h}$ e $K_{v} \circ$ coeficiente de difusividade turbulenta de calor e vapor de água, respectivamente; $\left(T_{1}-T_{2}\right)$ e $\left(e_{1}-e_{2}\right)$ são, respetivamente, gradiente de temperatura e umidade do ar na camada $\Delta \mathrm{z}=\mathrm{z}_{2}-\mathrm{z}_{1}$. A razão de BOWEN para atmosfera neutra carece do termo $\mathrm{K}_{\mathrm{h}} / \mathrm{K}_{\mathrm{v}}$ por que nestes casos estes coeficientes tem valores iguais, então:

$$
\beta=\gamma \frac{\left(T_{1}-T_{2}\right)}{\left(e_{1}-e_{2}\right)}
$$

No presente trabalho, durante o período diurno, não foi encon trada condição de neutralidade, pelo menos nas horas de leitu ra, no que se refere à atmosfera livre, isto $\bar{e}$, na camada $\Delta=142-20 \mathrm{~cm}$ acima do solo. Apesar desta situação $\mathrm{K}_{\mathrm{h}}$ diferente de $\mathrm{K}_{\mathrm{v}}$, foi decidido usar o método de balanço de energia, pelo fato de afirmar-se que o método é pouco sensivel às incorreções devidas aos coeficientes de difusividade turbulenta (CHANG, 1974). Usando a equação psicromētrica para $e_{1}$ e $e_{2}$ subs- 
tituindo na equação (9) (VILLA NOVA, 1973.), chega-se a:

$$
\beta=1 /\left[\frac{\Delta T_{h}}{\Delta T} \cdot\left(\frac{1}{\gamma} \cdot \frac{\Delta e_{s h}}{\Delta T_{h}}+1\right)-1\right]
$$

sendo $\Delta \mathrm{T}=$ gradiente de temperatura do ar na camada em consi deração; $\Delta \mathrm{T}_{\mathrm{h}}$ gradiente de temperatura do bulbo ümido na cama da em consideração; $\Delta e_{s h}=$ gradiente de pressão de saturação do bulbo molhado, também na camada em consideração; $\gamma=\mathrm{CpP} / \mathrm{LL}\left(\frac{\mathrm{m}_{\mathrm{b}}}{\mathrm{QK}_{\mathrm{K}}}\right)$, constante psicrométrica. Esta equação (10) é a utilizada neste trabalho.

3.2.2.2. Energia de evapotranspiração (LE)

Para sua quantificação foi utilizada a expres são:

$L E=\left(R_{n}-G\right) /(1+\beta)$

2.2.2.3. Energia de aquecimento do ar (H)

Ela foi calculada com a equação:

$H=\beta$. LE

3.2.3. Método aerodinâmico

3.2.3.1. Estabilidade atmosférica

pelo fato do método aerodinâmico não dar bons resultados em consideração ao princípio da similaridáde (PRUITT, 
1963; RIDER, 1954), no presente caso utilizou-se equações com termos de correção adiabạtica. Para isso foi necessārio ana lisar a estabilidade da atmosfera na camada em consideração; isto foi feito comparando o gradiente tērmico vertical (GT) com o gradiente adiabático seco (AG), onde:

$$
\mathrm{GT}=\frac{\mathrm{T}_{1}-\mathrm{T}_{2}}{\mathrm{z}_{2}-\mathrm{z}_{1}}\left(\frac{\mathrm{\varphi K}}{\mathrm{cm}}\right)
$$

sendo $T_{1}-T_{2}$ a variação térmica entre $z_{1}$ e $z_{2}$, sendo $z_{1}=20$ $\mathrm{cm} \mathrm{e} z_{2}=142 \mathrm{~cm}$ (ambos acima do solo). Para gradiente adia bático seco utilizou-se $A G=0,00010 \mathrm{~K} / \mathrm{cm}$. A comparação dos gradientes podem dar os seguintes casos:

a) $\mathrm{GT}=\mathrm{AG}$, condição de neutralidade

b) GT > AG , condição de atmosfera instável

c) GT < AG, condição de atmosfera estāvel.

3.2.3.2. Número de Richardson (Ri)

Ele é um parâmetro adimensional que expressa a razão entre a turbulência livre e a turbulência forçada, ou seja, razão entre forças convectivas térmicas e forças me cânicas de cisalhamento do vento. Além da estabilidade atmosférica, ele permite determinar o tipo de turbulência (PRIEST LEY, 1955), assim para $0>: R i>-0,02$ predomina turbulência forçada, para $\mathrm{Ri}>-0,03$ predomina a turbulência livre, correspondendo ao regime de transição valores entre $-0,02$ e $-0,03$. 
A equação utilizada é:

$$
R i=\frac{g\left(\frac{\Delta T}{\Delta z}+A G\right)}{\bar{T}\left(\frac{\Delta u}{\Delta z}\right)^{2}}
$$

sendo: $g=980 \mathrm{~cm} / \mathrm{s}^{2}$

$$
\begin{aligned}
\mathrm{AG}= & 0,0001^{\circ} \mathrm{K} / \mathrm{cm} \\
\overline{\mathrm{T}}= & \text { temperatura absoluta }\left({ }^{\circ} \mathrm{K}\right) \text { média da camada } \Delta \mathrm{z} \\
\Delta \mathrm{z}= & \text { camada do ar entre } 20 \text { e } 142 \mathrm{~cm} \text { acima do solo } \\
\Delta \mathrm{u}= & \text { variação de velocidade do vento }(\mathrm{cm} / \mathrm{s}) \text { na cama } \\
& \text { da em consideração }
\end{aligned}
$$

3.2.3.3. Função de Estabilidade $(\phi)$

Os termos de correção adiabática para os coeficientes de difusividade turbulenta de momento $\left(\mathrm{K}_{\mathrm{m}}\right)$, de vapor de água $\left(\mathrm{K}_{\mathrm{v}}\right)$ e de calor $\left(\mathrm{K}_{\mathrm{h}}\right)$ são $\phi_{\mathrm{m}}, \phi_{\mathrm{V}}$ e $\phi_{\mathrm{h}}$ respectivamente. Seus valores em condições de neutralidade são iguais a 1; para condições de instabilidade, menores que 1 e maiores que 1 para caso de condições estáveis (BAILEY \& DAVIES, 1981; THOM, 1975).

As funções de estabilidade para troca de momento $\left(\phi_{\mathrm{m}}\right)$, para vapor de água $\left(\phi_{\mathrm{v}}\right)$ e para calor $\left(\phi_{\mathrm{h}}\right)$, podem ser expressos como função do número de Richardson (BAILEY, 1981; THOM, 1975; VIEBB, 1970; MUNN, 1966 e LUMLEY, 1964), na seguinte forma:

para condições estáveis: $\phi_{\mathrm{V}}=\phi_{\mathrm{h}}=\phi_{\mathrm{m}}=(1-5 \mathrm{Ri})^{-1}$ 
para condições instāveis: $\phi_{\mathrm{V}}=\phi_{\mathrm{h}}=\phi_{\mathrm{m}}^{2}=(1-16 \mathrm{Ri})^{-1 / 2}$

e no caso de neutralidade: $\phi_{\mathrm{v}}=\phi_{\mathrm{h}}=\phi_{\mathrm{m}}=1$

\subsubsection{Energia de evapotranspiração (LE)}

Para sua estimativa segundo o método aerodinâa mico foi utilizada a equação:

$$
L E=-\left(\frac{\rho C_{p}}{\gamma}\right) \frac{k^{2}}{\phi_{m} \phi_{v}} \cdot \frac{\Delta e \cdot \Delta u}{\left(\operatorname{Ln}\left(\frac{z_{2}}{z_{1}}\right)\right)^{2}}
$$

onde: $\rho$ é a densidade do ar dada por

$$
\rho=\frac{P-e}{R d \cdot T}\left(\frac{g}{\mathrm{~cm}^{3}}\right)
$$

sendo P a pressão atmosférica (mb); e a pressão de vapor atual; T a temperatura do ar $\left({ }^{\circ} \mathrm{K}\right)$ e Rd a constante especifica do ar seco tendo os valores de:

$$
\begin{aligned}
& \mathrm{Rd}=2,8704 \times 10^{3} \frac{\mathrm{mb} \cdot \mathrm{cm}^{3}}{\mathrm{~g} \cdot \mathrm{O}_{\mathrm{K}}} \\
& \mathrm{Rd}=68,67 \times 10^{-3} \frac{\mathrm{cal}}{\mathrm{g} \cdot \mathrm{O}_{\mathrm{K}}} \\
& \mathrm{Rd}=2,1533 \times 10^{3} \frac{\mathrm{mmHg} \cdot \mathrm{cm}^{3}}{\mathrm{~g} \cdot \mathrm{O}_{\mathrm{K}}}
\end{aligned}
$$

$\mathrm{Cp}=0,24\left(\frac{\mathrm{cal}}{\mathrm{g} \cdot{ }^{\circ} \mathrm{K}}\right)$, calor específico do ar seco a pressão constante. 
$\gamma=\frac{c_{p} p}{\varepsilon L}\left(\frac{m b}{o_{K}}\right), \quad e ́$ a constante psicrométrica.

$\mathrm{K}=0,4$, constante de Von Karman.

$\phi_{\mathrm{m}}$ e $\phi_{\mathrm{v}^{\prime}}$ respectivamente, função de estabilidade para troca turbulenta de momento e de vapor de água.

$\Delta e=$ variação vertical da umidade do ar (mb) entre os niveis $z_{1}$ e $z_{2}$.

$\Delta \mathrm{u}=$ variação vertical da velocidade do vento $(\mathrm{m} / \mathrm{s})$ na camada $z_{2}-z_{1}$.

Considerando os valores das constantes e a equação (18) e efetuando as conversões a equação (17) fica como:

$$
L E=-2,18 \times 10^{-4}\left(\frac{p-e}{\mathrm{~T}}\right) \frac{\mathrm{L}}{\phi_{\mathrm{m}} \mathrm{v}_{\mathrm{v}}} \frac{\Delta_{\mathrm{e}} \cdot \Delta \mathrm{u}}{\left(\mathrm{In} \frac{\mathrm{z}_{2}}{\mathrm{z}_{1}}\right)^{2}}\left(\frac{\mathrm{cal}}{\mathrm{cm}^{2} \cdot \min }\right)
$$

onde L é o calor latente de mudança de estado de água líquido a vapor e seu valor está dado pela equação $(6)$; ( $\Delta$ e devem ser dados em mb e $\mathrm{T}$ temperatura média $\left({ }^{\circ} \mathrm{K}\right)$ da camada do ar em consideração.

3.2.3.5. Energia de aquecimento do ar (H)

Para a estimativa do fluxo vertical da energia de aquecimento do ar ou calor sensível, segundo este método, foi utilizada a equação: 


$$
\mathrm{H}=\frac{\rho \mathrm{C}_{\mathrm{p}} \mathrm{K}^{2}}{\phi_{\mathrm{m}} \phi_{\mathrm{h}}} \frac{\Delta \theta \cdot \Delta \mathrm{u}}{\left(\operatorname{Ln} \frac{\mathrm{z}_{2}}{\mathrm{z}_{1}}\right)^{2}}
$$

onde: $\rho=$ densidade do ar seco $\left(\mathrm{g} / \mathrm{cm}^{3}\right)$

$$
\begin{aligned}
\stackrel{\mathrm{C}}{\mathrm{C}}= & \text { calor específico a pressão constante do ar seco } \\
\mathrm{K}= & \text { constante de von Karman } \\
\phi_{\mathrm{m}} \text { e } \phi_{\mathrm{h}=} & \text { função de estabilidade de troca turbulenta de } \\
& \text { momento e de calor sensivel. } \\
\Delta \mathrm{u}= & \text { variação de velocidade do vento entre os niveis } \\
& \mathrm{z}_{2} \text { e } \mathrm{z}_{1}(\mathrm{~m} / \mathrm{s}) \\
\Delta \theta= & \operatorname{variação~de~temperatura~potencial~entre~os~niveis~} \mathrm{z}_{2} \text { e } \mathrm{z}_{1}(\mathrm{OK})
\end{aligned}
$$

A variação $(\Delta \tilde{\theta})$ da temperatura potencial entre os niveis $z_{1}$ e $z_{2}$ é dada por:

$$
\Delta \theta=\left(\mathrm{T}_{1}-\mathrm{T}_{2}\right)\left(\frac{1000}{\mathrm{P}}\right)^{0,288}
$$

levando em conta que a pressão atmosférica média para abril e maio em Piracicaba é igual a $955.52 \mathrm{mb}$ a expressão se transforma em:

$$
\Delta \theta=1,0132\left(\mathrm{~T}_{1}-\mathrm{T}_{2}\right)
$$

Considerando as equações (18) e (22) e substituindo os valores das constantes em(20) obtemos:

$$
\mathrm{H}=8,133 \times 10^{-2}\left(\frac{\mathrm{P}-\mathrm{e}}{\mathrm{T}}\right) \frac{1}{\phi_{\mathrm{m}} \phi_{\mathrm{h}}} \frac{\left(\mathrm{T}_{1}-\mathrm{T}_{2}\right)\left(\mathrm{u}_{2}-\mathrm{u}_{1}\right)}{\left(\mathrm{Ln} \frac{\mathrm{z}_{2}}{\mathrm{z}_{1}}\right)^{2}} \frac{\mathrm{cal}}{\mathrm{cm}^{2} \cdot \min }
$$


onde: $T=$ temperatura absoluta média $(O K)$ entre os niveis $z_{1}$ $\mathrm{z}_{2}$.

$\left(\mathrm{T}_{1}-\mathrm{T}_{2}\right)$ e $\left(\mathrm{u}_{2}-\mathrm{u}_{1}\right)$ são, respectivamente, as variações térmica e de velocidade do vento entre os níveis $z_{1}$ e $z_{2}$

$P$ e 'e' são pressão atmosférica média do local e pressão de vapor de água, respectivamente.

No presente estudo os dados de temperatura $(T)$, umidade (e) foram dos niveis de 20 e $80 \mathrm{~cm}$, isto é, $z_{2}=80 \mathrm{~cm}$ e $z_{1}=20 \mathrm{~cm}$ mas os ventos $u_{1}$ e $u_{2}$ foram de níveis de 40 e 100 $\mathrm{cm}$ respectivamente.

3.2.4. Coeficiente de transferência de vapor de āgua (Dv)

3.2.4.1. Quantificação

Considerando o fluxo vertical médio da ener gía de evapotranspiração de uma superfície de água ou superfície bem irrigada dado por:

$$
L E_{O}=-\left(\frac{\rho C_{p}}{\gamma}\right) \frac{d e}{d z}\left(k_{v}+k_{v}\right)
$$

e integrando esta equação desde a superfície evaporante $\left(z_{1}=0\right)$ até outro nível $\left(\mathrm{z}_{2}\right)$, que geralmente é $2 \mathrm{~m}$ acima da superficie, temos:

$$
L E_{0}=\left(\frac{\rho C_{p}}{\gamma}\right)\left(e_{0}-e_{z}\right)\left(\int_{0}^{z} \frac{1}{k_{v}+K_{v}} d z\right)^{-1}
$$


e fazendo que

$$
D v=\left(\int_{0}^{z} \frac{1}{k_{v}+k_{v}} d z\right)^{-1}
$$

então:

$$
L E_{0}=\left(\frac{\rho C_{p}}{\gamma}\right) D_{v}\left(e_{o}-e_{z}\right)
$$

Segundo SELLERS (1967) e BUDYKo (1956), a equação (25) permite determinar o coeficiente de transferência de vapor de água (Dv), sendo necessário para isso medir a evaporação de superfícies aquáticas ou bem irrigadas.

Considerando a equação (18), a constante psicrométrica $\gamma=\mathrm{C}_{\mathrm{p}} \mathrm{P} / \mathrm{EL}$ e substiturndo as demais constantes, $\mathrm{Ob}$ temos de (25):

$$
D V=77,104 \frac{T \cdot L E_{O}}{L\left(e_{O}-e_{z}\right)}\left(\frac{\mathrm{cm}}{\mathrm{s}}\right)
$$

onde $\mathrm{T}$ = temperatura média do ar da camada em consideração (OK)

LE ${ }^{\prime}=$ evapotranspiração de uma superfî́cie bem irrigada ou evaporação de uma superfície de água $\left(\mathrm{cal} / \mathrm{cm}^{2}\right.$. $\operatorname{min)}$.

$L=$ calor latente de evaporação (cal/g) dada pela equação (6).

$\left(e_{0}-e_{z}\right)=$ variação vertical da umidade do ar (mb), entre a superfície e o nível z. 
Neste estudo as variações $\Delta z$ utilizadas no mētodo de balanço de energia for am para as camadas:
a) $40-80 \mathrm{~cm}$
b) $20-80$
C) $20-142 \mathrm{~cm}$
d) $40-142 \mathrm{~cm}$
e) $10-142 \mathrm{~cm}$
f) $0-142 \mathrm{~cm}$

Para o método aerodinâmico foram ensaiadas as camadas de:
a) $20 \mathrm{~cm}-80 \mathrm{~cm}$
b) $40 \mathrm{~cm}-142 \mathrm{~cm}$

Todas estas camadas foram ensaiadas, porque, neste trabalho, tínhamos uma camada evapotranspirante e não uma superfície evaporante. Além disso a evapotranspiração (LE) não fói só calculada para uma superfície bem irrigada, mas também foi para caso mais geral, incluindo todos os casos.

\subsubsection{Relações empíricas para estimativas de Dv}

procuraram-se dois tipos de relações empirricas: a) relação linear simples com a velocidade do vento a dois metros de altura, e b) relação linear múltipla com dados de abrigo meteorológico. 


\subsection{Relação linear simples}

Foi ensaiada a relação linear sugerida por

SELLERS (1967) dada pela equação

$$
\mathrm{DV}=\mathrm{a}+\mathrm{bu}
$$

utilizando-se a velocidade do vento medida a $2 \mathrm{~m}$ de altura. o interesse é obter uma equação geral representativa para o perî́odo total de experimento. o procedimento seguido foi o de buscar as relações para as observações diārias e selecionar aqueles onde o teste estatístico fosse significativo. Nos dias selecionados fazer a homogeneização dos dados por simples ins peção, isto ê, comparando o conjunto de pares de dados $D v$ e $V$ do dia, prescindir daqueles onde valores pequenos do Dv estiver associados a vento intenso ou vice-versa. Finalmente correlacionar todo o conjunto de dados.

\subsection{Relações lineares múltiplas}

Como nem sempre se possui dados de vento, necessārio estabelecer equações empíricas que o relacionem a dados que mais frequentemente são medidos nos postos meteorológicos, tais como temperatura, temperatura máxima, temperatú ra mínima, umidade relativa, etc. Procurou-se relações com três variāveis indenpendentes da forma:

$$
D v=a+b_{1} x_{1}+b_{2} x_{2}+b_{3} x_{3}
$$

nos seguintes casos: 

a) $D v=a+b_{1} T+b_{2} T m+b_{3} \Delta T$
b) $D v=a+b_{1} T+b_{2} T n+b_{3} \Delta T$
c) $\mathrm{Dv}=\mathrm{a}+\mathrm{b}_{1} \mathrm{~T}+\mathrm{b}_{2} \mathrm{RU}+\mathrm{b}_{3} \Delta \mathrm{T}$

sendo $\mathrm{T}$ a temperatura do ar; Tm a temperatura mínima; $\Delta \mathrm{T}$ a di ferença entre temperatura máxima e mínima; Tn a temperatura máxima e UR a umidade relativa. Os dados de temperatura, umi dade relativa e DV foram dados médios para o dia.

\subsubsection{Tratamento dos dados de vento}

Devido à algumas irregularidades no registro do vento nos níveis de 40,100 e $160 \mathrm{~cm}$, alguns dados correspondentes às leituras no início e final do dia foram perdidas.

Para solucionar tal problema e conseguir uma série de dados de vento mais completa, os dados de 10 observa ções, foram estimados.

Para isto considerou-se que a relação entre o vento a $2 \mathrm{~m}$ de altura e os outros níveis inferiores è dada pela expressão:

$$
\mathrm{Uz}=\mathrm{Uh}\left(\frac{\mathrm{z}}{\mathrm{h}}\right)^{\mathrm{a}}
$$

onde: $\mathrm{Uz}=$ velocidade do vento a nível desejado $(z)$.

$\mathrm{Uh}=$ velocidade do vento a $2 \mathrm{~m}$ de altura $(\mathrm{h})$.

$\mathrm{a}$ = uma constante. 
Para usar a equação (29), nos dias com dados perdidos, foi necessário determinar, previamente, o valor da constante a; o qual foi realizada mediante uma regressão linear simples entre LnUz e LnUh, expressa como:

$$
\operatorname{LnUz}=b_{0}+b_{1} \text { LnUh }
$$

onde o termo $b_{0}$ inclui a constante a na forma da seguinte expressão:

$$
a=b_{0} / \operatorname{Ln}(z / h)
$$




\section{RESULTADOS E DISCUSSÃO}

\subsection{Evapotranspiracão}

Este elemento é um dos parâmetros muito impor tantes na quantificação do coeficiente de transferência do vapor de água, sendo um dos componentes que indiretamente afeta as magnitudes das contantes ' $a$ ' $e$ ' $b$ ' na equação empírica en tre Dv e vento. Os alcances ou as limitações de qualquer equação empírica a ser deduzida utilizando Dv, é função direta dos elementos que the deram origem; razão pela qual é necessário discutir previamente os parâmetros que afetam seu valor. Como é conhecido, a.lém do gradiente da pressão de vapor, LE é um elemento decisivo em sua quantificação; a diferença deste LE com $\Delta$ e é que o $\Delta$ e é medido experimentalmen te e LE é uma variável estimada. Daí a razão que precisar se ter mais cuidado com sua medida.

ivo presente trabalho, IE foi estimado por dois méto dos, o balanço de energia e o aerodinâmico. As estimativas foram feitas para cada hora de observação resultando 10 esti mativas por dia, com um total de 23 dias para método de ba- 
37.

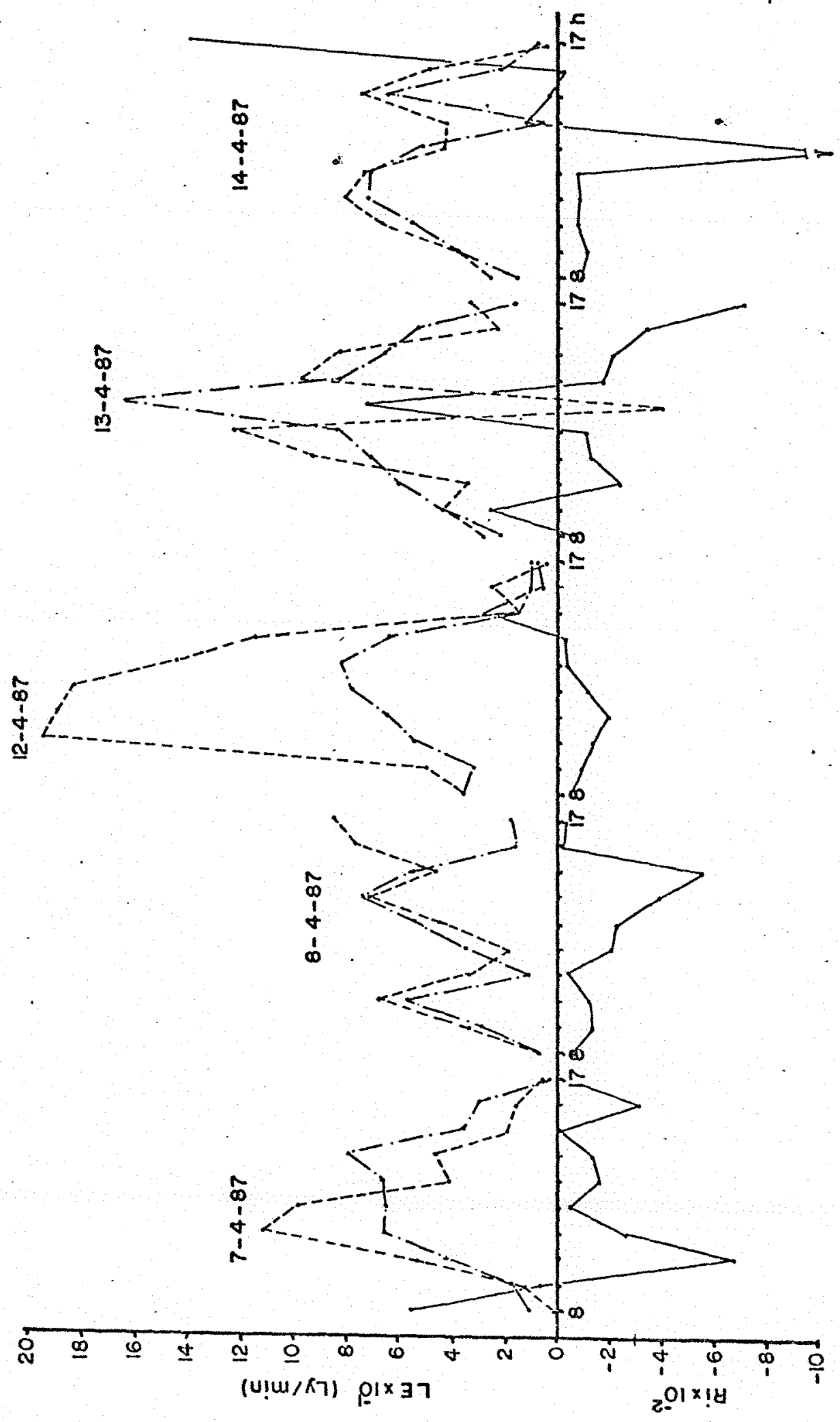

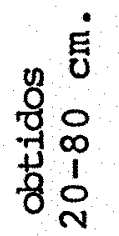

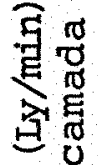

团

18

U.

4

2

I

8

80

क.

4

$-8$

78

I.

कि 0

\&

y

얼

मे

ช

0 व

용

' 8

8 든

$\pi \pi$

त.

10

용

i⿱

wis

त्र

is

莺 


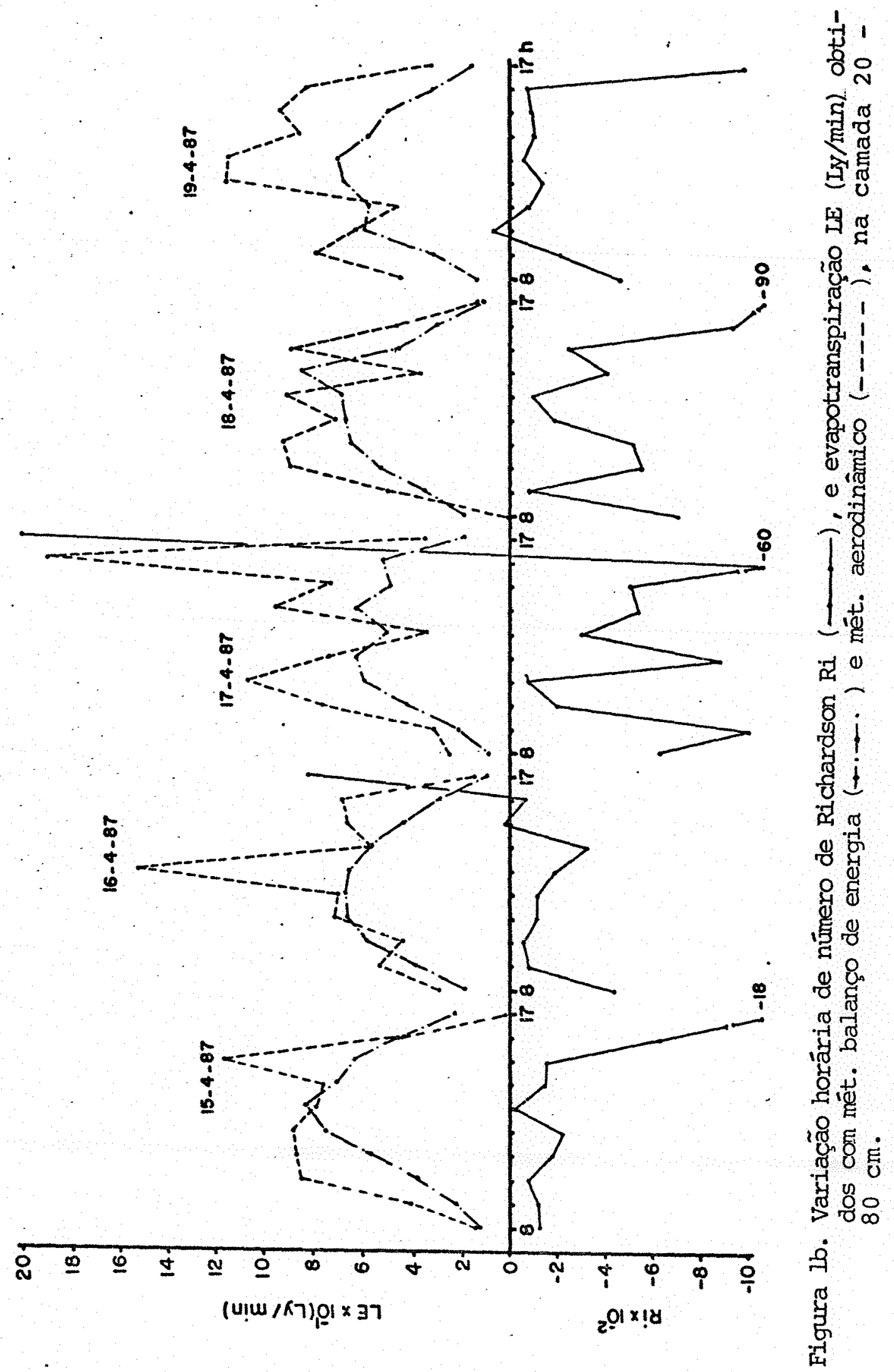


lanço de energia (M.B.E.) e 19 dias para o método aerodinâmico (M.A.). Para a presente análise foram tomados 10 dias entre todos, que são apresentados em forma gráfica nas Figuras la e lb; as quais mostram a variação horāria do nümero de Richardson Ri, evapotranspiração LE tanto para M.B.E. como para M.A., para a camada $20-80 \mathrm{~cm}$. A Tabela 1 apresenta os dados diários de radiação líquida $R n$, fluzo de calor no solo $G$ e vento médio a 40,100 e $200 \mathrm{~cm}$ de altura. Na Tabela 2 podem ser observados os valores obtidos para evapotranspiração LE, calor sensível H e os valores médios diários do coeficiente de transferência DV.

A análise das Figuras la e lb, para o caso de LE, indica que na maioria dos casos a evapotranspiração correspondente ao M.A. è sempre maior daqueles correspondentes ao M.B.E. A diferença é maior nos dias com vento intenso, como pode ser visto no dia 12/04/87. Segundo a Tabela 1 verificase que este foi um dos dias com vento mais intenso e, ainda, segundo a Tabela 2 , verifica-se que LE do M.B.E. foi $46 \%$ de LE do M.A. Similar efeito no M.A. exerce a variação vertical da umidade do ar $(\Delta e)$ nos dias $15,16,17$ e 19 de abril (Figu ra 1b). Os aumentos súbitos de LE são devidos aos valores al tos de $\Delta e$. A diferença mais marcante entre os dois métodos ocorre nos dias frios, com nuvens e com vento intenso. Estas situações predominam nos últimos dias da observação, que pode-se verificar pelo sinal do fluxo de calor sensível H, onde houve diferenças de 100\%. Podemos dizer que o M.A. superesti 
TABELA 1 Totais diarios de Radiacáo Liquida Rn (cal/cmz.dia), Fluxo do calor no solo G (cal/cm².dia) e vento medio $V(\mathrm{~m} / \mathrm{s})$ a 40,100 e $200 \mathrm{~cm}$ de altura.

\begin{tabular}{|c|c|c|c|c|c|}
\hline Data & Rn & G & $V_{40}$ & $V_{100}$ & $V_{200}$ \\
\hline \multicolumn{6}{|l|}{ Abril } \\
\hline 6 & 281.3 & 14.66 & - & - & 1.82 \\
\hline 7 & 359.9 & 22.38 & 0.72 & 1.16 & 1.72 \\
\hline$\theta$ & 267.2 & 15.61 & 0.67 & 1.30 & 2.08 \\
\hline 12 & 307.5 & 14.09 & 0.76 & 1.54 & 2.33 \\
\hline 13 & 387.1 & 20.34 & 0.30 & 0.95 & 1.30 \\
\hline 14 & 254.2 & 10.68 & 0.94 & 1.67 & 2.17 \\
\hline 15 & 341.8 & 21.59 & 0.32 & 0.95 & 1.38 \\
\hline 16 & 333.5 & 24.63 & 0.36 & 1.02 & 1.71 \\
\hline 17 & 322,2 & 26.77 & 0.33 & 0.79 & 1.35 \\
\hline 18 & 345.4 & 26.41 & 0.26 & 0.66 & 1.22 \\
\hline 19 & 332.0 & 26.05 & 0.49 & 1.27 & 1.89 \\
\hline 20 & 120.1 & -2.14 & 1.13 & 1.87 & 2.78 \\
\hline 25 & 247.0 & 19.21 & - & - & 3.19 \\
\hline 26 & 301.1 & 16.46 & 0.86 & 1.82 & 2.66 \\
\hline 27 & 249.6 & 18,63 & 0.76 & 2.11 & 3.78 \\
\hline \multicolumn{6}{|l|}{ Maio } \\
\hline 2 & 151.2 & 11.30 & - & - & 1.57 \\
\hline 3 & 295.6 & 13.54 & - & - & 1.56 \\
\hline 7 & 171.6 & 9.84 & 0.30 & 1.20 & 2.42 \\
\hline $\mathrm{g}$ & 134.5 & 1.32 & 0.92 & 2.45 & 3.69 \\
\hline 10 & 211.9 & 9.34 & 1.33 & 2.32 & 3.48 \\
\hline 13 & 295.3 & 18.68 & 0.35 & 1.19 & 1.92 \\
\hline 14 & 219.7 & 11,07 & 0.62 & 1.84 & 2.75 \\
\hline 28 & 50.87 & 4.52 & 0.15 & 0.66 & 1.09 \\
\hline
\end{tabular}


TABELA 2 Valores diarios de evapotranspiraço LE (mm/dia), calor sensivel $H(c a l / c m=$ dia) e valores media diaria do coeficinte de transferencia Dv(cm/s)

\begin{tabular}{|c|c|c|c|c|c|c|c|c|c|}
\hline \multirow{3}{*}{ at: } & \multicolumn{6}{|c|}{ Metodo de balango de energia } & \multicolumn{3}{|c|}{ Met. Aeorodinatmico } \\
\hline & \multicolumn{3}{|c|}{ Camada $20-80=m$. } & \multicolumn{3}{|c|}{ Camada $20-142 \mathrm{~cm}$. } & Camada & \multicolumn{2}{|c|}{$20-80 \mathrm{~cm}}$. \\
\hline & LE & $\mathrm{H}$ & Dv & LE & $H$ & Dv & LE & H & DV \\
\hline \multicolumn{10}{|l|}{ Abril } \\
\hline 6 & 3.56 & 53.84 & .38 & 3.82 & 37.16 & 6.86 & - & - & - \\
\hline 7 & 4.17 & 88.62 & & 4.10 & 122.3 & & 4.13 & 81.31 & 4.96 \\
\hline $\mathbf{B}$ & 3.55 & 39.85 & 5.06 & 3.34 & 56.60 & 5. & 4.89 & 48.85 & 6.93 \\
\hline 12 & 4.51 & 3.69 & 3.69 & 4.51 & 25.24 & 5 & 71 & 54.60 & .65 \\
\hline $1:$ & 6. & -39.9 & 3. & 4.90 & -20 & 8. & & 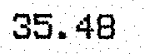 & 52 \\
\hline 14 & 4. & 0.2 & 5 & 3 & 4. & & & 2 & 6.37 \\
\hline 15 & 4.92 & 26 & 4. & 4. & 29. & & & 42 & 5.86 \\
\hline 16 & 4.52 & 38.62 & 5.21 & 4.24 & 63.21 & 5. & 8 & 54.90 & 5.41 \\
\hline 17 & 4.24 & 42.47 & 3.82 & 4.25 & 40. & 3. & 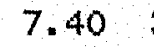 & 3. & 6.04 \\
\hline 18 & 4.69 & 39.04 & 4. & $4.8 E$ & 53. & & & 5 & 4.85 \\
\hline 19 & 4 & 38 & & 2 & & & & & 7.45 \\
\hline 20 & 1. & 16.98 & 4. & 1 & 11 & 3 & 2.64 & 30.06 & 6.37 \\
\hline 25 & 3.55 & 15.99 & 3.57 & 3.50 & 19.11 & 3.00 & - & - & - \\
\hline 26 & 4.69 & 4.61 & 5.70 & 4.54 & 12,67 & 4.62 & 7.49 & 10.7 & 7.85 \\
\hline 2 & 3.56 & 18.25 & 5.04 & 3.49 & 23.80 & 3.93 & 8.38 & 15.84 & 10.8 \\
\hline \multicolumn{10}{|l|}{ Mai } \\
\hline 2 & 2.65 & -18 & 3.37 & 4 & -1 & & - & - & - \\
\hline 3 & 5.08 & -21.2 & 7.31 & 4.75 & -3.69 & E. & - & - & - \\
\hline 7 & 3.10 & -23.0 & 5.49 & 3.08 & -17.8 & 4.87 & 3.08 & -52.9 & 8.35 \\
\hline$B$ & 3.14 & -54.3 & 9.23 & 3.08 & -54.1 & $6.6 E$ & .93 & -95.9 & 12.3 \\
\hline 1 & 3.01 & 22.74 & 3.86 & 2.99 & 25.31 & & .56 & 34.02 & 8.95 \\
\hline & 3.99 & 38.24 & 6.08 & 3.31 & 30,69 & 4.85 & 3.57 & 46.98 & 8.69 \\
\hline 14 & 3.56 & -3.73 & 6.10 & 3.55 & -3.12 & 3.78 & 5.33 & -20.3 & 10.12 \\
\hline 28 & 0.54 & 14.07 & 3.31 & 0.74 & 1.76 & 2.76 & 0.97 & 19.14 & 3.99 \\
\hline
\end{tabular}


ma valores de LE. E óbvio que cada um dos métodos tem suas limitações. E assim que no M.B.E. considerou-se o princípio da similaridade e no M.A. a função estabilidade $(\phi)$.

No que se refere ao princípio da similaridade, este mostrou-se válido aproximadamente em $90 \%$ dos dias analisados e, em média, em $60 \%$ das horas de observação. Isto pode ser observado nas figuras la e $1 \mathrm{~b}$, analisando o número de Richardson. Observa-se nelas que houve maior número de horas com Ri entre 0 e $-3 \times 10^{-2}$, o que é um argumento bom para afirmar que as estimativas do M.B.E. foram as mais adequadas.

Os dois métodos fracassaram em situações de adevcção de ar mais úmido, nas quais $\Delta e=e_{1}-e_{2}$, era negativo; dando valores muito elevados no M.B.E. e valores negativos no M.A. Esta situação observa-se em 13 de abril às 13:00 horas.

\subsection{Coeficiente de transferência DV}

Na Tabela 2 apresentam-se os valores médios de Dv para as camadas $20-80 \mathrm{~cm}$ e $20-142 \mathrm{~cm}$ no M.B.E. e para as camadas $20-80 \mathrm{~cm}$ no M.A. As camadas apresentadas são aquelas nas quais foram encontradas correlações significativas entre Dv e $V$ em um maior número de dias. Os valores horā rios oscilaram entre 0,4 e $17 \mathrm{~cm} / \mathrm{s}$ no M.B.E. e entre 0,1 a 17 $\mathrm{cm} / \mathrm{s}$ no M.A. Como no caso da evapotranspiração, em geral, maiores valores foram obtidos no M.A. A oscilação horária de Dv do M.A. pode ser observada nas Figuras $2 a, 2 b$ e 2c. 


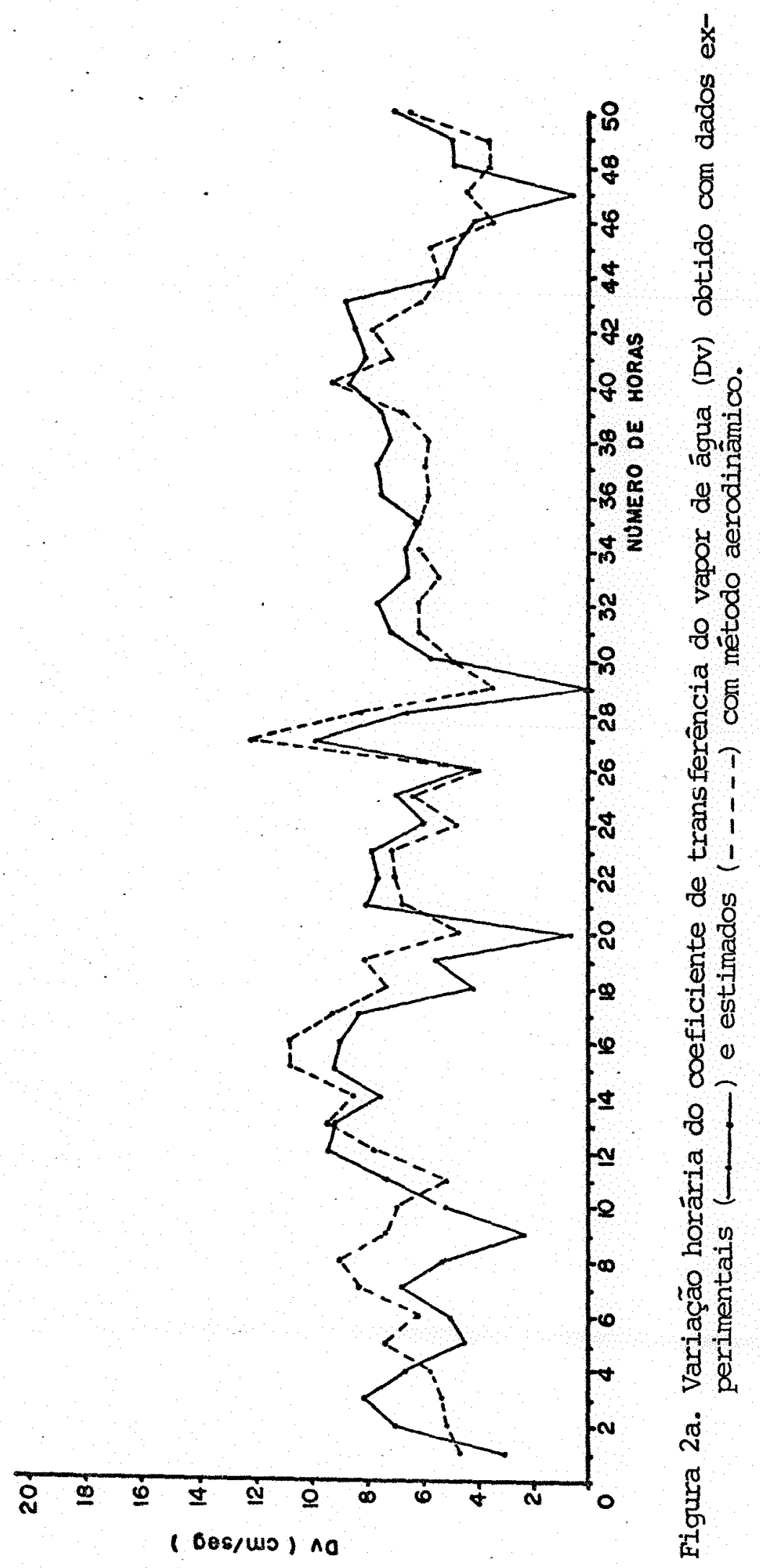




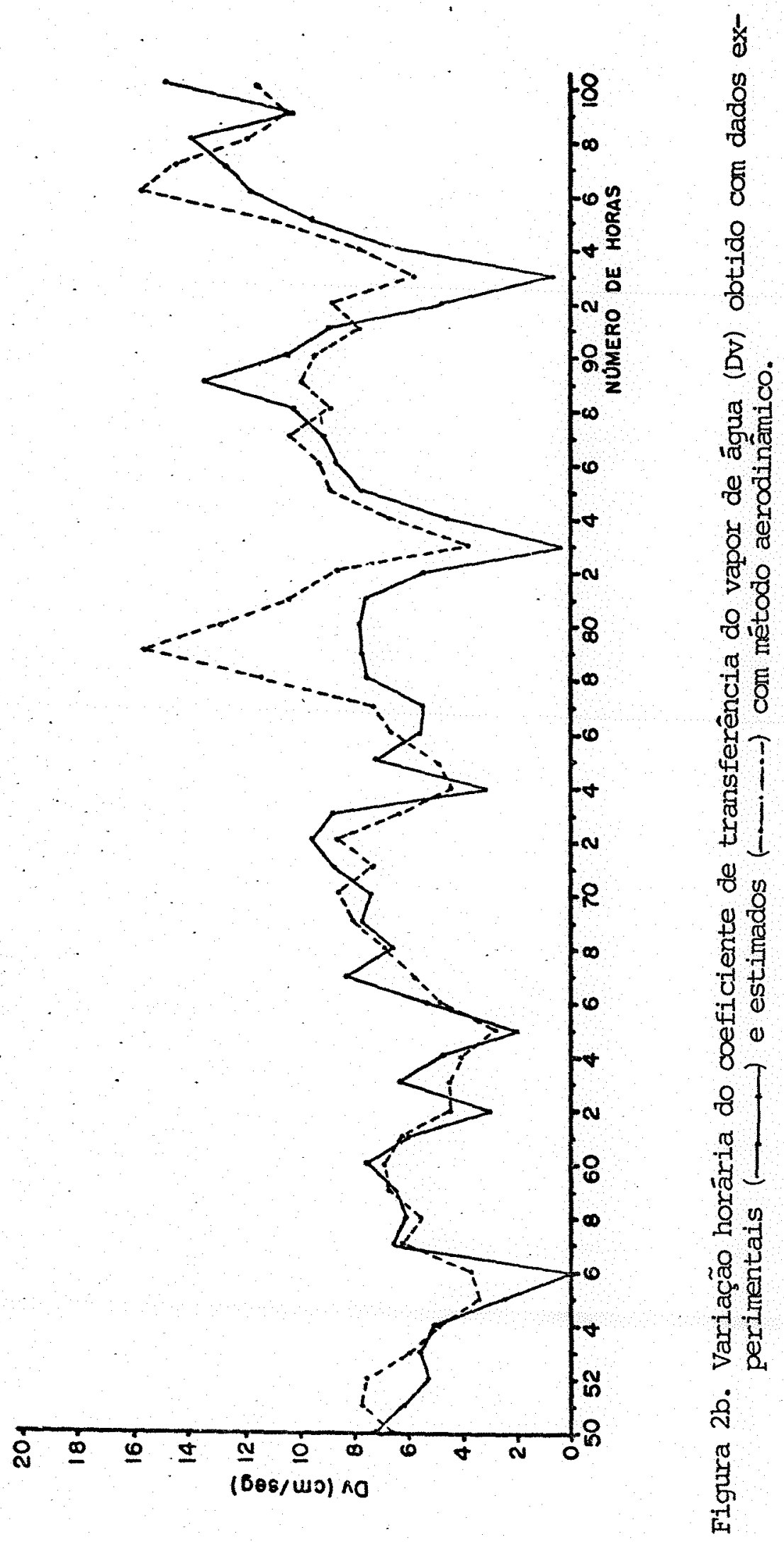




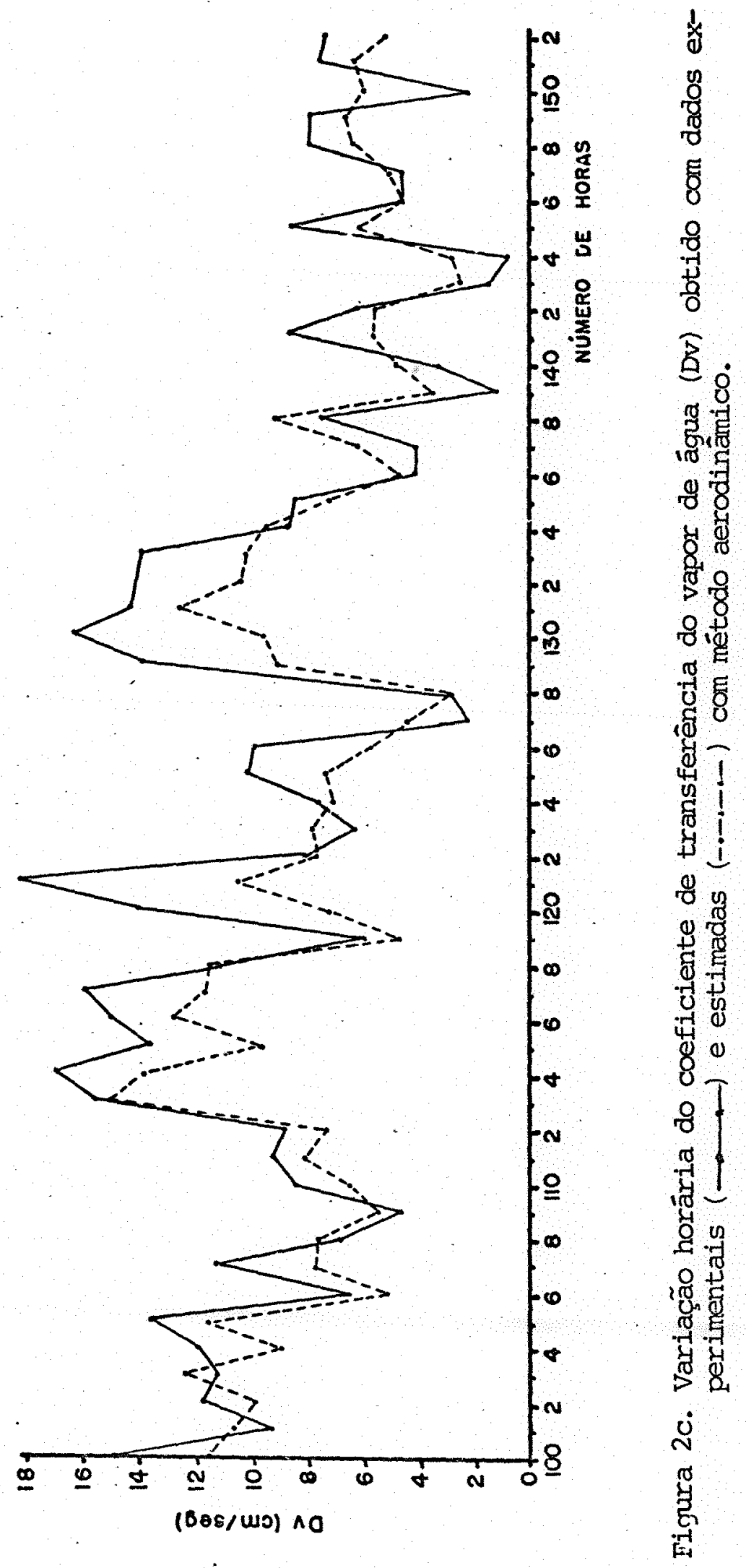


Pelo fato de ter-se considerado uma camada situada a $20 \mathrm{~cm}$ da superfície, pode-se desprezar a componente molecular no processo de transferência vertical. Ainda, considerando que Kv é independente da altura z nesta camada de $60 \mathrm{~cm}$, seu valor pode ser dado por:

$$
\mathrm{KV}=\mathrm{DV} \Delta \mathrm{z}=60 \mathrm{DV}
$$

e de acordo com os valores extremos de Dv. indicados previamente, vemos que $\mathrm{kv}$ oscilou entre $6 \mathrm{e} 1.020 \mathrm{~cm}^{2} / \mathrm{s}$, que são va lores razoāveis e se admitirmos que $\mathrm{Kv}=\mathrm{Kh}$, estes valores es tão dentro daqueles determinados por VILLA NOVA (1973) que apresenta valores de $\mathrm{Kh}$ oscilando entre $220 \mathrm{e} 1.550 \mathrm{~cm}^{2} / \mathrm{s}$, em cujo caso a camada de $30 \mathrm{~cm}$ dituou-se $50 \mathrm{~cm}$ acima da superficie com cultura de arroz. Observa-se, nos dois casos, usando metodologias distintas, conseguiu-se valores de coeficientes de difusividade turbulenta kv que estão nas mesmas ordens de grandeza, fato que indica dois dados de Dv obtidos no referido trabalho, estar dentro de variações que podem ocorrer na prātica.

4.3. RELAcoes eMPiRI ICAS PARA ESTIMATIVA DE DV

\subsubsection{Relação linear simples}

Com os dados de DV e de vento $V$ registrados a $2 \mathrm{~m}$ de altura procurou-se relações entre eles para cada dia de observação, fazendo uso da equação (27). Para o M.B.E., en controu-se correlações significativas em 13 dias para camada 
20-80 cm e em 15 dias para a camada 20-142 cm. Parao M.A. 17 dias forneceram correlações significativas. Após realizar a análise de consistência dos dados nos dias em que as correlações foram significativas, fez-se a anālise de correlação geral para todos os dias. Os resultados obtidos encontram-se nas Tabelas 3 e 4 para $O$ M.B.E e na Tabela 5 para $O$ M.A. Observa-se que no M.B.E. os testes foram altamente significativos para as duas camadas consideradas. Mas analisando as magnitudes do coeficiente de correlação e de F calculado(FC), vemos que os melhores resultados foram os correspondentes à camada $20-80 \mathrm{~cm}$. Da mesma forma o resultado estatístico cor respondente ao M.A. foi altamente significativo, com um coefí ciente de correlação $(0,76)$ e FC muito maiores que no outro método. Por outro lado, analisando os erros relativos médios (ER), aparentemente as equações do M.B.E. são as melhores, com um erro aproximado de $54 \%$ em ambos os casos, conte $55 \%$ do M.A. Mesmo assim, a magnitude dos très erros é muito alto - que nos obriga a analisar sua variabilidade dentro da amostra utilizada. Tal análise foi realizada comparando os DV quantificados com dados experimentais e os estimados com a equação empírica estabel ecida, que para o caso do M.A. apresen tou maior erro. Isto è apresentado nas figuras $2 \mathrm{a}, 2 \mathrm{~b}$ e $2 \mathrm{c}$. Da observação destas figuras, podemos afirmar que a equação obtida é de boa precisão e que o valor alto do erro é consequência de que, em certos dias, na primeira ou última horas do dia, ocorrem superestimativas. Isto pode ser observado nos horários $20,29,47,56,83$ e 93. São estes valores os 
TABELA 3: Quadro da anal ise da variancia para M. B. E. (20-80cm.)

\begin{tabular}{|c|c|c|c|c|c|}
\hline Fonte & $S C$ & GL & $\mathrm{CM}$ & $F$ & $\mathrm{RF}$ \\
\hline Regressato & 191.1112 & 1 & 191.1112 & 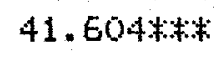 & .2607 \\
\hline Erro & 542.0438 & 118 & 4.5936 & & \\
\hline Total & 733.155 & 119 & & & \\
\hline
\end{tabular}

*** : Signif, ao nivel.99 林 : Signif. ao nivel.95

$Y=2.582+1.238 x \quad r=.5106 \quad F C=41.604 \%$ EF $(\%)=54.76$

TABELA 4: Quadro da analise da variAncia para M. B. E. $(20-142 \mathrm{~cm}$ )

\begin{tabular}{llllll}
\hline Fonte & SC & GL & CM & F & FR \\
\hline Regressato & 118.1906 & 1 & 118.1906 & 21.561 *a. & .1491 \\
Erro & 674.2549 & 123 & 5.4817 & & \\
Total & 792.4455 & 124 & & &
\end{tabular}

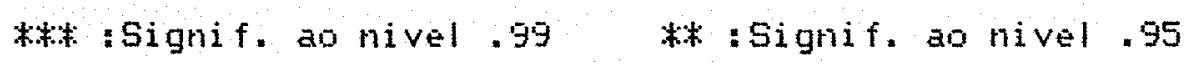

$Y=2.568+.947 X \quad r=.3662 \quad F E=21.561 * \alpha \% \quad E R(\%)=53.86$

TABELA 5: Guadro da analise da variancia para M. A. $(20$ - Bon.m)

\begin{tabular}{llllll}
\hline Fonte & SC & GL & CM & F & RR \\
\hline Regres530 & 1182.4935 & 1 & 1182.4935 & 205.83 w. & .5736 \\
Erro & 878.9861 & 153 & 5.745 & & \\
Total & 2061.4796 & 154 & & & \\
\hline
\end{tabular}

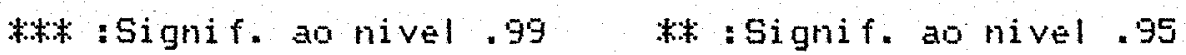

$Y=2.069+2.455 X \quad r=.7574 \quad F E=205.83 \% * \quad E R(\%)=54.96$ 
que contribuem para valores altos do ero relativo médio. Ain da podemos dizer que o valor alto do erro è resultado de superestimativa, por realizar-se o quociente de números muito pequenos.

\subsubsection{Relações lineares múltiplas}

Com valores médios diārios de Dv ensaiaram-se correlações múltiplas, de acordo com a equação (28). Tanto no M.B.E. como no M.A., as variáveis independentes foram tem peratura $(T)$, temperatura mínima $(\mathrm{Tm})$, temperatura máxima (TM), amplitude térmica diurna $(\Delta T)$ e a umidade relativa (UR), todos dados do abrigo meteorológico. Apresentam-se, na Tabe la 6 , só os resultados que foram significativos ou aqueles onde o Fc estivesse próximo ao F crítico, representado por F $(90 \% ; 3,11)=2,61$ no caso do M.B.E. e $F(90 \%, 3,9)=2,81$ no caso do M.A.

Observa-se na tabela mencionada, que O M.B.E. não houve equações que foram significativas; de todos os casos ensaiados, o modelo que teve como variáveis independentes $T$, UR e $\Delta$ T, foi o melhor e com o Fc próximo a F crítico ou tabular. O mesmo modelo no caso de M.A. resultou significativo. A comparação dos valores quantificados com dados experimentais com os estimados, que pode observar na Figura 3 , indicam que as estimativas não diferem em denasia dos originais. 
TABELA 6 Relacoes empiricas multiplas para estimativa do coeficiente de transferencia do vapor de ugua DV

Metodo de balanfo de energia:

Equacao planteado $D V=a+b 1 T+b 2 U R+b 3 A T$

Eq. encontrada $Y=0,9356+0,1926 X_{1}+0,0332 X_{2}-0,2053 X_{3}$

Parametros estatisticos $F C=2,1 \quad R^{2}=0,36 \quad \operatorname{Er}(\%)=18,6$

$$
F(90 \% ; 3,12)=2,61
$$

Metodo aerodinamico:

$$
\begin{aligned}
& \text { 1. } \quad \text { DV }=a+b_{I} T+b_{2} T_{M}+b_{9 A T} \\
& Y=10,09-6,004 X_{1}+0,303 X_{z}-0,929 X_{3} \\
& \text { Fc }=2,64 \quad R^{2}=0,47 \quad \operatorname{Er}(\%)=21,3 \\
& F(90 \%, 3,9)=2.81 \\
& \text { 2. }-\quad D_{V}=a+b_{1} T+b_{2} U R+b_{3} A T \\
& Y=30.622+0.0452 T-0.1573 \mathrm{UR}-0.9978 \mathrm{AT} \\
& F C=3,71 * \quad R^{2}=0,55 \quad \operatorname{Er}(\%)=17,7 \\
& F(90 \% ; 3,9)=2,81
\end{aligned}
$$



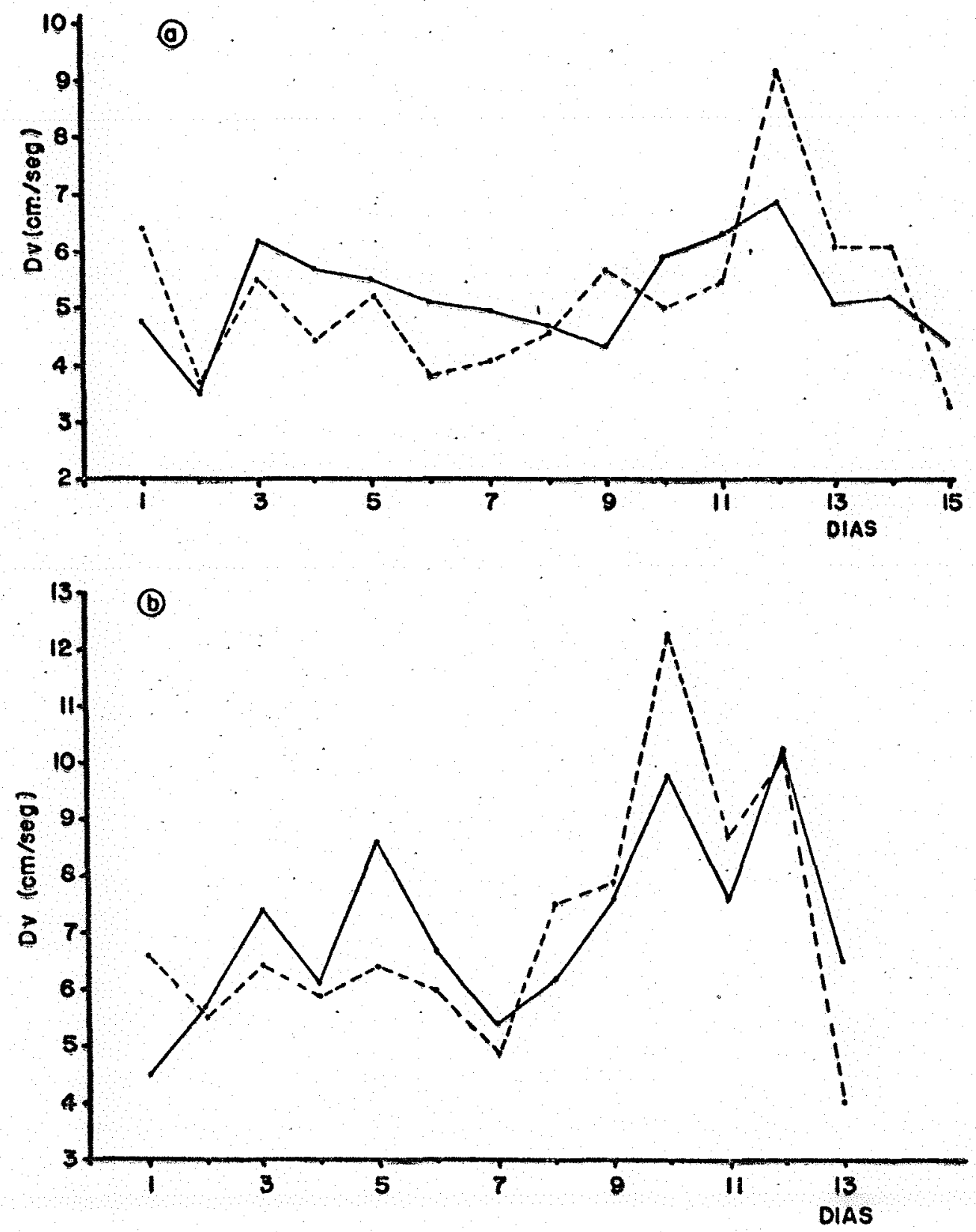

Figura 3. Valores médios diários do coeficiente de transferên cia do vapor de água (Dv) obtidos com dados experi mentais (--b--) ) e estimados $(\longrightarrow-)$ com mëtodo de balanço de energia (a) e mëtodo aerodinâmico (b). 


\section{CONCLUSÕES}

A interpretação e discussão dos resultados con duzem às seguintes conclusões:

1. Na estimativa da evapotranspiração, os valo res obtidos com M.B.E. foram menores que os do M.A.; essas di ferenças em dias frios, com cèu coberto e vento forte è bem marcada, onde O LE do M.B.E. pode ser até 100\% menor do que do M.A.

2. Os valores dos coeficientes de transferência vertical do vapor de água (Dv) quantificados estiveram dentro dos limites de $0,4-17 \mathrm{~cm} / \mathrm{s}$. Aqueles correspondentes a M.A. foram maiores dos M.B.E., mas as diferenças foram pequenas.

3. As relações empíricas, lineares simples, entre Dv e o vento $V$, foram altamente significativas para os dois mêtodos; mas os valores de Fc e $\gamma$ indicaram maior grau de associação do vento com Dv correspondente ao método aerodi nâmico. 
4. As relações empíricas, lineares múltiplas, foram melhores quando feitas com temperatura (T), umidade relativa (UR) e a amplitude tērmica diurna (AT), como variāveis independentes; mas estatisticamente só foram significativas no caso do M.A. 


\section{LITERATURA CITADA}

ANDRE, R.G.B., 1981. Um estudo dos transportes verticais de momentum, calor sensível e vapor d'água sobre superfície vegetada nos trópicos. São José dos Campos, INPE / CNPq, 112p. (Tese de Doutoramento).

BAILEY, W.G. e J.A. DAVIES, 1981. The effect of uncertainly in aerodynamic resistence on evaporation estimates from the combination models. Boundary-Layer Meterology, 20: 187-199.

BUDYKo, M.I., 1956. Teplovoi balans Zemnoi poverkhnosti, Le ningrad, Gidrometerologichskoe isdatel' stvo; Translated as the Heat Balance of the Earth's surface by Nina stepa nova. U.S. Weather Bureau, Dept. of Commerce, 1958.

CHANG, J.H., 1974. Climate and agriculture. Aldine publishing Company/Chicago.

COCHEME E FRANQUIN, 1967. A study of agraclimatology of the semiarid area south of the Sahara in West Africa.

DEACON, E.L.; C.H.B. PRIESTLY e W.C. SWINBANK, 1958. Evaporation and water balance. In: Climatology. UNESO. p.934. 
FRITSCHEN, L.J. e GAY, L.W. Environmental instrumentation. New York, Springer-Verlag, 1979. 215p.

GARCIA, V.J. 1984a. Estimación del termino a ero dinamico de la evapotrasnpiracion. (Aceito para sua publicação na revista - Anales Científicos UNA).

GARCIA, V.J., 1984b. Fundamento teórico de la ecuación de evapotranspiración potencial de Penamn. Cuadernos de físí ca y Meteorología. Vol. IX, no 22.

GILBERT, M.J. e C.H.M. VAN BAVEL, 1954. A simple field method for measuring maximum evapotrasnpiration. Transaction. American Geophysical Union, 35: 937-42.

HALTINER, G.M., 1957. Dynamical and physical meteorology. New York, MCGraw-Hill Book Co.

LUMLEY, J.L. e H.A. PANOFSKY, 1964. The stricture of atmospheric turbulence. New York, John Wiley.

MONTHEITH, J.L., 1973. Principles of environmental physics. London, Edward Arnold. $241 \mathrm{p}$.

MUNN, R.E., 1966. Descriptive micrometeorology. New York, Acaḍemic Press.

OLDEMAN, L. A FRERRE, M., 1982. A study of agroclimatology of humid tropics of South East Asia. WMO Technical Note, no 179 .

OMETTO ( J.C., 1981. Bioclimatologia vegetal. São paulo, Agronômica Ceres Ltda. 425 p. 
PASQUILL, J.C., 1950. Some further consideration of the measurement and indirect evaluation of natural evapotranspira tion. Quaterly Journal, Royal Meteorological Society, 76: 287-301.

PENMAN, H.L., 1956. Estimating evaporation; Transaction. American Geophysical Union, 37:43-50.

PRIESTLEY, C.H.B., 1955. Free and forcedconvection in the atmosphere mear the ground. Quaterly Journal, Royal Meteo rology society, 81: $139-43$.

PRUIT, W.0., 1963. Application of several energy balance and aerodynamic evaporation equations under a wide range of stability. Davis, University of California.

RANZANI, G.0.; O. FREIRE; T. KINJO, 1966. Carta do solo do município de Piracicaba. Piracicaba, ESALQ/USP.

RIDER, N.E., Eddy diffusion of momentum, water vapor, and heat near ground. Philosophical Transaction, Royal Society Series A, 246: $481-501$.

ROSENBERG, N.J., 1974. Microclimate: the biological environment. New York, John Wiley Sons. $315 \mathrm{p}$.

SELLERS, W.D., 1967. Physical climatology. Chicago, University of Chicago Press. 272 p.

SMITHONIAN, I.P., 1984. Smithsonian meteorological tables. washington, Smithonian Institution Press.

STANHILI, G., 1962. The use of Piche evaporimeter in the cal culation of evapotranspiration. Quaterly Journal, Royal Meteorological Society, 88: 80-2. 
SUTTON, O., 1953. Micrometeorology. MgGraw-Hill Book Company. New York.

TANNER, C.B., 1960. Energy balance approach to evapotranspiration from crops. Proceedings. Soil Science Society of America, 24: 1-9.

TANNER, C.B., 1963. Energy relations in plant community, En vironment control of plant growth. New York, Academic Press.

THOM, A.S., 1975. Momentum, mass and heat exchange of plant communities. In: MONTEITH, J.L. Vegetation and the atmos phere. London, Academic Press, p.57-109.

THONTHWAITE, C.W., 1965. The loss of water to the air. Meteorological Monographs. University of London, London.

VILLA NOVA, N.A., 1973. Estudos sobre o balanço de energia em cultura de arroz. Piracicaba, ESALQ/USP. 89p. (Tese de Livre-Docência).

YAMAMOTO, G., 1975. Generalization of the kEYPES formula in diabatic conditions and related discussion on the criti cal Richardson number. J. Meter. Soc. Japan, 53: 189-95.

WEBB, E.K., 1970. Profile relationships: the log - linear range, and extension to strong stability. Quart. Journal Royal Meteorological Society, 9667-70. 
58.

\section{APENDICE}

\section{CONJUNTO DE TERMÔMETROS DE TERMISTORS}

a. - Circuito do conjnto dos termômetros

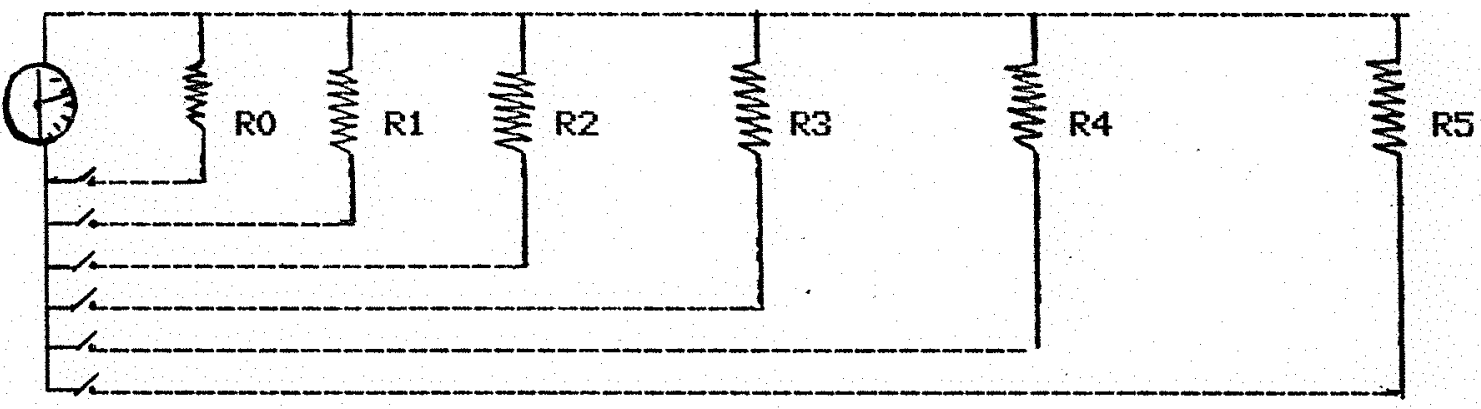

b.- Esquena do conjunto dos termômetros

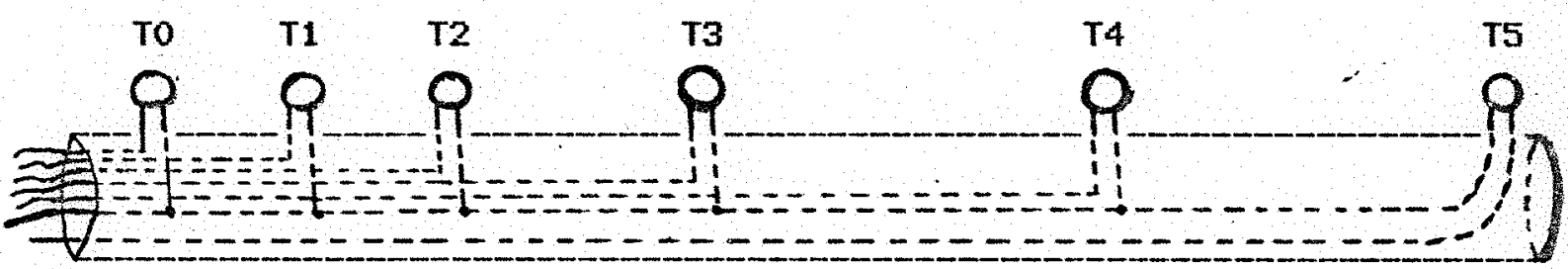

To: Termômetro ou termistor 0 , T1: Termômetro ou termistor $1, \ldots$, T5: Termônetro ou termistor 5 . 